\title{
A statistical model for the prediction of wind-speed probabilities in the atmospheric surface layer
}

Article

Accepted Version

Efthimiou, G. C., Hertwig, D., Andronopoulos, S., Bartzis, J. G. and Coceal, O. (2017) A statistical model for the prediction of wind-speed probabilities in the atmospheric surface layer. Boundary-Layer Meteorology, 163 (2). pp. 179-201. ISSN 0006-8314 doi: https://doi.org/10.1007/s10546-016-0221-2 Available at https://centaur.reading.ac.uk/68026/

It is advisable to refer to the publisher's version if you intend to cite from the work. See Guidance on citing.

To link to this article DOI: http://dx.doi.org/10.1007/s10546-016-0221-2

Publisher: Springer

All outputs in CentAUR are protected by Intellectual Property Rights law, including copyright law. Copyright and IPR is retained by the creators or other copyright holders. Terms and conditions for use of this material are defined in the End User Agreement.

www.reading.ac.uk/centaur 
Central Archive at the University of Reading

Reading's research outputs online 


\title{
A statistical model for the prediction of wind-speed probabilities in the atmospheric surface layer
}

\author{
G. C. Efthimiou ${ }^{\text {la }}$, D. Hertwig ${ }^{\mathrm{b}}$, S. Andronopoulos ${ }^{\mathrm{a}}$, J.G. Bartzis ${ }^{\mathrm{c}}$, O. Coceal ${ }^{\mathrm{d}}$ \\ ${ }^{a}$ Environmental Research Laboratory, INRASTES, NCSR Demokritos, Patriarchou Grigoriou \\ $\&$ Neapoleos Str., 15310, Aghia Paraskevi, Greece. \\ ${ }^{b}$ Department of Meteorology, University of Reading, Reading, UK. \\ 'University of Western Macedonia, Dept. of Mechanical Engineering, Sialvera \& Bakola Str., \\ 50100, Kozani, Greece. \\ ${ }^{\mathrm{d}}$ National Centre for Atmospheric Science, Department of Meteorology, University of \\ Reading, Reading, UK.
}

\begin{abstract}
Wind fields in the atmospheric surface layer (ASL) are highly three-dimensional and characterized by strong spatial and temporal variability. For various applications such as wind comfort assessments and structural design, an understanding of potentially hazardous wind extremes is important. Statistical models are designed to facilitate conclusions about the occurrence probability of wind speeds based on the knowledge of low-order flow statistics. Being particularly interested in the upper tail regions we show that the statistical behavior of near-surface wind speeds is adequately represented by the Beta distribution. By using the properties of the Beta probability density function in combination with a model for estimating extreme values based on readily available turbulence statistics, it is demonstrated that this novel modelling approach reliably predicts the upper margins of encountered wind speeds. The model's basic parameter is derived from three substantially different calibrating datasets of flow in the ASL originating from boundary-layer wind-tunnel measurements and direct numerical simulation. Evaluating the model based on independent field observations of nearsurface wind speeds showed a high level of agreement between the statistically modelled horizontal wind speeds and measurements. The results show that, based on the knowledge of only a few simple flow statistics (mean wind speed, wind speed fluctuations and integral time scales), the occurrence probability of velocity magnitudes at arbitrary flow locations in the ASL can be estimated with a high degree of confidence.
\end{abstract}

\section{Keywords}

Atmospheric surface layer; Beta distribution; Direct numerical simulation; Extreme wind speeds; Field experiment; Probability density function; Weibull distribution; Wind tunnel

\section{Introduction}

\footnotetext{
${ }^{1}$ Corresponding author. Tel: +30 2106503405. E-mail address: gefthimiou@ipta.demokritos.gr (G. C. Efthimiou)
} 
Knowledge of the probability density function (p.d.f.) of wind speed in the atmospheric boundary layer (ABL) is necessary for many applications such as the estimation of the wind energy potential at a particular location (Sarkar et al., 2011) or wind comfort and safety studies (e.g. Janssen et al., 2014). Furthermore, safety considerations in the design of structures at exposed locations (e.g. bridges, radio masts or wind turbines) require a reliable assessment of the maximum expected wind speeds (Brabson and Palutikof 2000).

For the prediction of wind gusts, i.e. sudden, brief increases of local wind speeds, various methodologies have been proposed in the past. Zhang et al. (2013), for example, performed an analysis of the characteristics of $1-\mathrm{Hz}$ wind-speed data continuously sampled in the air layer below $2 \mathrm{~m}$. They proposed an empirical model to predict the fluctuating wind gusts of the streamwise velocity based on friction velocity, mean wind speed and standard deviation at $2 \mathrm{~m}$. Sallis et al. (2011) used a machine-learning approach to determine meaningful and robust results of wind gusts and proposed an algorithm for application to real-time climate data. Brasseur (2001) proposed a new wind gust estimate method where the determination of gusts is fully based on physical considerations. The proposed approach assumes that surface gusts result from the deflection of air parcels in the upper levels of the boundary layer, which are brought down by turbulent eddies. The method takes into account the mean wind and the turbulent structure of the atmosphere.

Over the past years, research activity in the area of wind-speed distribution modelling has increased considerably. For the prediction of wind-speed distributions statistical models that provide information about the local occurrence probabilities at a certain site are preferably employed. For this task, the choice of a suitable p.d.f. is crucial. A number of previous studies compared statistical distributions with measurements in order to examine how well the p.d.f.s describe the statistical properties of the measured wind speed. An overview of recent studies is presented in Table 1 .

Table 1. Overview of recent studies (in chronological order) that use different statistical distributions to assess wind speed occurrence probabilities.

\begin{tabular}{|l|l|l|}
\hline Publication & Distributions & $\begin{array}{l}\text { Averaging time interval of data } \\
\text { analyzed }\end{array}$ \\
\hline Donk et al. (2005) & Weibull & $1 \mathrm{~h}$ \\
\hline Carta et al. (2009) & $\begin{array}{l}\text { Generalized Gamma, Gamma, } \\
\text { Weibull, singly truncated from } \\
\text { below normal, two components } \\
\text { mixture Weibull, Rayleigh, beta, } \\
\text { inverse Gaussian, lognormal }\end{array}$ & $1 \mathrm{~h}$ \\
\hline He et al. (2010) & Weibull & $1 \mathrm{~h}$ \\
\hline Morgan et al. (2011) & $\begin{array}{l}\text { Gamma, lognormal, Rayleigh, log } \\
\text { Pearson type III, Generalized } \\
\text { Rayleigh, Generalized Gamma, } \\
\text { Pearson type III, Weibull, } \\
\text { Generalized normal, Wakeby, }\end{array}$ & \\
\hline
\end{tabular}




\begin{tabular}{|l|l|l|}
\hline & Kappa, bimodal Weibull mixture & \\
\hline Waewsak et al. (2011) & Weibull & $10 \mathrm{~min}$ \\
\hline Aidan (2011) & $\begin{array}{l}\text { Normal, Gamma, Weibull, } \\
\text { Rayleigh }\end{array}$ & 1 month \\
\hline Sarkar et al. (2011) & $\begin{array}{l}\text { Weibull, extreme value distribution } \\
\text { of type I (Gumbel) }\end{array}$ & $1 \mathrm{~h}$ \\
\hline Odo et al. (2012) & Weibull & $1 \mathrm{~d}$ \\
\hline Kollu et al. (2012) & $\begin{array}{l}\text { Weibull-extreme value distribution } \\
\text { (GEV), Weibull-lognormal, GEV- } \\
\text { lognormal }\end{array}$ & $10 \mathrm{~min}$ \\
\hline Masseran et al. (2013) & $\begin{array}{l}\text { Lognormal, Weibull, Rayleigh, } \\
\text { exponential, Gamma, inverse } \\
\text { Gaussian, Burr, inverse Gamma }\end{array}$ & $1 \mathrm{~h}$ \\
\hline Carneiro et al. (2016) & Weibull, exponentiated Weibull & $1 \mathrm{a}$ \\
\hline Nemeş (2013) & Weibull & $1 \mathrm{~h}$ \\
\hline Indhumathy et al. (2014) & Weibull & $1 \mathrm{~h}$ \\
\hline Kidmo et al. (2015) & Weibull & $1 \mathrm{~h}$ \\
\hline Metković (2015) & Weibull al (2016) & - \\
\hline
\end{tabular}

Table 1 shows that a large number of different p.d.f.s were previously compared with wind speed data, with the Weibull distribution overall being the most popular choice.

In order to assess extreme wind speeds, extreme value theory can be used (e.g. Palutikof et al. 65 1999; Holmes and Moriarty 1999; Simiu et al. 2001). However, in this case, the successful modelling of the upper tail can often lead to an inadequate representation of the main part of

67 the wind-speed distribution. Most of the studies listed in Table 1 used 1-h or 10-min 68 averages. Steinkohl et al. (2010) analyzed the wind-speed measurements on a finer time scale 69 in the so-called micrometeorological range. Their dataset consisted of observations measured 70 in the ABL on two different days with a sampling frequency of $1 \mathrm{~Hz}$. They focused on the modelling of the tail of the wind-speed distribution by using the 'peaks-over threshold' 72 approach of extreme value theory.

73 Other studies use nonparametric estimators of wind speed. Rozas-Larraondo et al. (2014), for 74 example, studied a new method based on nonparametric multivariate locally weighted 
regression for improving wind speeds forecast by a numerical weather prediction model. Wind direction data were used to build different regression models, as a way of accounting for the effect of surrounding topography. Recently, D'Amico et al. (2014) presented a new nonparametric model to predict wind speeds based on semi-Markov chains. They found the model to be able to reproduce the statistical behavior of wind speeds accurately for different time scales when used as a forecast tool. Francisco-Fernández and Quintela-del-Río (2013) applied nonparametric curve estimation methods to analyze time series of wind speeds, focusing on extreme events exceeding a chosen threshold. Nonparametric methods to directly estimate quantities such as the probability of exceedance, the quantiles or return levels or the return periods were proposed. Moreover, bootstrap techniques were used to develop pointwise and simultaneous confidence intervals for these functions.

\subsection{Aim of this study}

In this study we aim at demonstrating that occurrence probabilities of wind speeds in the atmospheric surface layer (ASL) can be estimated based on the knowledge of low-order flow statistics readily available from field measurements and the choice of a p.d.f. that has a finite range. In quantitative terms we aim to predict the horizontal wind speed $V$ within the time interval $\Delta \tau$ that is encountered at an arbitrary location,

$V(\Delta \tau)=\frac{1}{\Delta \tau} \int_{\Delta \tau} v(t) d t$,

where $v(t)$ is the instantaneous wind speed. $V(\Delta \tau)$ is in meteorological terminology the $\Delta \tau$ gust.

The time interval $\Delta \tau$ can signify a measurement time interval or an averaging period, as is the case in this study. However, $\Delta \tau$ can be assigned further significance when associating it with typical exposure times to certain wind speeds, e.g. based on the time an individual is expected to stay at a particular location. While mean velocities in urban areas are typically low, turbulence levels can be significant and wind gusts can by far exceed the time-averaged wind speeds encountered at street level, which can cause discomfort for pedestrians or result in structural damage to buildings. The time scales associated with such gust episodes are as low as times associated with typical pedestrian walking speeds (i.e. a few s).

The derivation of a new p.d.f. for $V(\Delta \tau)$ and the associated maximum expected wind speed $V_{\max }(\Delta \tau)$ will be the main effort in the present investigation. A suitable p.d.f. should satisfy the following criteria:

1. It should describe all parts of the wind-speed distribution accurately.

2. It should have a finite upper extreme.

3. It should be applicable to a wide range of turbulent flows in the ASL.

One probability distribution that fulfils these criteria is the Beta distribution. 
110 The methodology employed in this study is described in detail in the next section. The

111 experimental and numerical wind speed databases are introduced in Sect. 3. Based on this 112 calibrating data, in Sect. 4 the basic parameter of the proposed model is estimated. Finally, in 113 Sect. 5 the methodology is validated based on independent field data.

114

115

116

117

118

119

132

\section{Methodology}

The present methodology is based on the studies by Bartzis et al. (2008) and Bartzis et al. (2015), in which the cumulative distribution function (c.d.f.) of scalar concentrations from point source releases of airborne materials was modelled. In the present study we apply the same methodology to the prediction of wind speed p.d.f.s and c.d.f.s.

For this, we make the hypothesis that the maximum wind speed that can be encountered in the ASL takes a finite value and that this maximum wind speed $V_{\max }(\Delta \tau)$ can be predicted by the Bartzis et al. (2008) model. We present a detailed justification for the applicability of the model in the following.

\subsection{Probability density function selection and parameterization}

It is assumed that the p.d.f. for the time-averaged horizontal wind speed $V(\Delta \tau)$ at a certain location is given by the Beta distribution (e.g. Gupta and Nadarajah, 2004)

$p d f(x) \propto x^{\alpha-1}(1-x)^{\xi-1} ; \quad 0 \leq x \leq 1$.

In the case of our study $x$ is given by a normalized form

$x=\frac{V(\Delta \tau)}{V_{\max }(\Delta \tau)}$,

and hence $x$ ranges from 0 to 1 .

The exponents $\alpha$ and $\xi$ are estimated from the wind speed mean, variance and the maximum value based on the general relationships for Beta distributions,

$$
\alpha=\frac{1}{1+\eta}\left(\frac{\eta}{\mathrm{I}}-1\right)
$$

$\xi=\eta \alpha$

$\eta=\frac{V_{\max }(\Delta \tau)-\bar{V}}{\bar{V}}$

where $V_{\max }(\Delta \tau)$ is the maximum time-averaged wind speed in the interval $\Delta \tau$ or in meteorological terminology the maximum $\Delta \tau$ gust in a large ensemble or a long time series. We obtain $V_{\max }(\Delta \tau)$ from the model proposed by Bartzis et al. (2008) (see Sect. 2.2), $\bar{V}$ is the mean wind speed and $I$ is the wind speed fluctuation intensity given by 
$139 \quad I=\frac{\sigma_{V}^{2}}{\bar{V}^{2}}$

140 with

$141 \sigma_{V}^{2}=\overline{V^{\prime 2}}$

142 where $\sigma_{V}^{2}$ is the variance and $V^{\prime}$ is the fluctuation, which are quantities that are routinely 143 available from different types of atmospheric flow models by solving the relevant equations 144 (e.g. Hertwig et al., 2012; Koutsourakis et al., 2012) or from experimental measurements. 145 Hence, in order to use the model the extreme value $V_{\max }(\Delta \tau)$ needs to be estimated. This is 146 described below.

\section{$147 \quad 2.2$ Extreme value analysis}

148 When adopting any finite range p.d.f. for the wind speed $V(\Delta \tau)$, the ability to estimate the 149 extreme value $V_{\max }(\Delta \tau)$ forms a prerequisite. It should be noted that the experimental 150 maximum $V_{\max }^{\text {meas }}(\Delta \tau)$ cannot be a priori expected to be the "true" extreme value as a direct 151 consequence of statistical uncertainties associated with limited measuring (or simulation) 152 times. For this reason we use the theoretical approach proposed by Bartzis et al. (2008) in 153 order to approximate the expected ("true") $V_{\max }(\Delta \tau)$, which is modelled by

$V_{\max }(\Delta \tau)=\bar{V}\left[1+b\left(\frac{\Delta \tau}{T_{V}}\right)^{-v} I\right]$

155 where $T_{V}$ is the wind speed integral time scale derived from the wind speed autocorrelation 156 function $R_{\nu}(\tau)$ via

$157 \quad T_{V}=\int_{0}^{\infty} R_{V}(\tau) d \tau$

and $R_{h}(\tau)$ is defined as

$159 \quad R_{V}(\tau)=\frac{\overline{V^{\prime}(t) V^{\prime}(t+\tau)}}{\overline{V^{\prime 2}}}$.

160 Eq. 5 was developed initially for the estimation of maximum concentrations of airborne 161 pollutants released from point sources. The theoretical background for the development of 162 Eq. 5 is based on the application of the following equation for a stationary time series of 163 infinite length:

$164 \frac{V_{\max }(\Delta \tau)}{V_{\max }(\Delta T)}=\left(\frac{\Delta \tau}{\Delta \mathrm{T}}\right)^{-n}$ 
Eq. 7 is used for the calculation of the maximum expected wind speed in a time interval $\Delta \tau$ when the maximum wind speed in a time interval $\Delta T$ is known. It follows a similar functional form as proposed by Bartzis et al. (2008) for concentrations. Following the same argument presented in Bartzis et al. (2008), here referring to wind speed instead of concentration, Eq. 7 is applied in deriving Eq. 5 based on the following assumptions:

(1) The maximum wind speed $V_{\max }(\Delta T)$ tends to the mean wind speed $\bar{V}$ as the time interval $\Delta T$ increases.

(2) The time interval by which the wind speed $V_{\max }(\Delta T)$ approximates $\bar{V}$ is analogous to the integral time scale of the wind speed $T_{V}$ (Eq. 6a).

(3) When the time interval $\Delta \tau$ increases, the wind speed $V_{\max }(\Delta \tau)$ approximates zero according to Eq. 7, while it should tend to $\bar{V}$. This accounts for the additional term of unity in Eq. 5.

(4) The ratio $V_{\max }(\Delta T) / \bar{V}$ depends on the fluctuation intensity $I$ (Eq. 4a).

Under these considerations the parameters that determine the extreme wind speed at a certain location are the fluctuation intensity $I$ and the integral time scale $T_{V}$, together with the constants $b$ and $v$.

The relationship presented in Eq. 5 was previously used successfully to predict maximum time-averaged pollutant concentrations from near-ground emission sources based on numerical results from simulations performed with computational fluid dynamics (CFD) models (Efthimiou and Bartzis 2011, 2014; Efthimiou et al. 2011a, 2011b, 2015). The parameters $b$ and $v$ in Eq. 5 can be derived empirically and typically exhibit a wide range of values as demonstrated in previous studies. This is a result of the combination of limitations of the model, experimental errors, insufficient stationarity of the time series and the finite duration of the analyzed signal used to derive these values. Previous studies on the dispersion of airborne material in atmospheric flows suggested indicative values of $b=1.5$ and $v=0.3$.

Several previous studies of wind gusts in the ASL have demonstrated that local gusts scale with the standard deviation of wind speed observed at the site (e.g. Beljaars 1987; Kristensen 1991), a parameter which indirectly includes information of surface roughness characteristics and effects of atmospheric stratification. This dependence is also included in the model shown in Eq. 5, via the fluctuation intensity $I$. Further information about the temporal scale of the phenomena is added by including a direct link to the local auto-correlation time scale $T_{V}$. The rationale for adopting the Bartzis et al. (2008) concentration model for the prediction of wind speed extremes (Eq. 5) is further based on the following:

1. The wind speed and the concentration are scalars, real numbers and take positive values.

2. There is relation between the wind and the concentration which is expressed through the Schmidt number.

3. It is expected that both variables have finite extreme values in the ABL.

4. The assumptions that were mentioned before for the construction of Bartzis et al. (2008) model are also considered to be valid for the wind speed. 
The last point is examined by analyzing sample data. For this purpose a random wind speed time series was selected from one of the experimental test cases used in this study (BL3-0 case described in Sect. 3.1.1.), which represents measurements in a rough-wall boundary layer modelled in the wind tunnel.

The first assumption is: "The maximum wind speed $V_{\max }(\Delta T)$ tends to be equal to $\bar{V}$ as the time interval $\Delta T$ increases." The maximum time-averaged wind speed $V_{\max }(\Delta T)$ of the random wind speed time series is plotted against $\Delta T$ in Fig. 1. Also the mean velocity is presented. The horizontal axis is plotted in logarithmic scale. It is clear that $V_{\max }(\Delta T)$ approximates $\bar{V}$ with the increase of $\Delta T$.

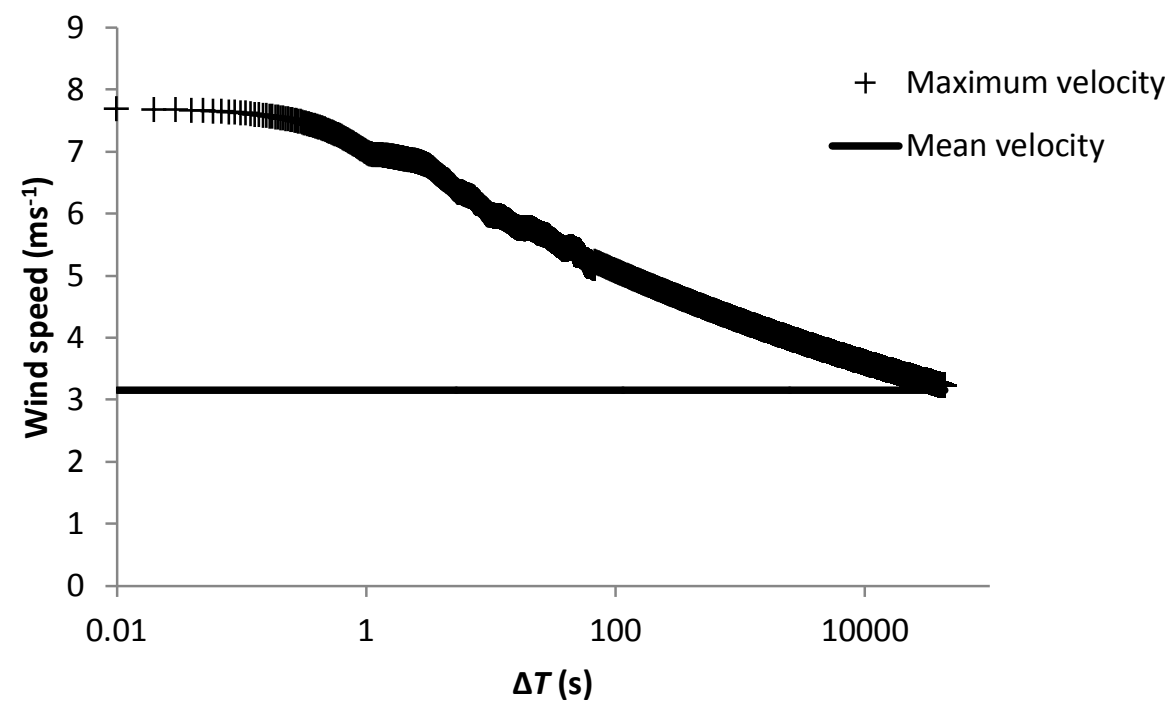

Fig 1 The maximum time-averaged wind speed and the mean wind speed are plotted versus the time interval $\Delta T$. The results correspond to a random wind speed time series of the BL3-0 case.

The second assumption is: "The time interval by which the wind speed $V_{\max }(\Delta T)$ approximates $\bar{V}$ is analogous to the integral time scale of the wind speed $T_{V}$ (Eq. 6a)." The ratio $V_{\max }(\Delta T) / \bar{V}$ of the random wind speed time series is plotted against $\Delta T / T_{V}$ in Fig. 2. There is a clear correlation $\left(R^{2}=0.9992\right)$ and a power-law function fits the data very well. 


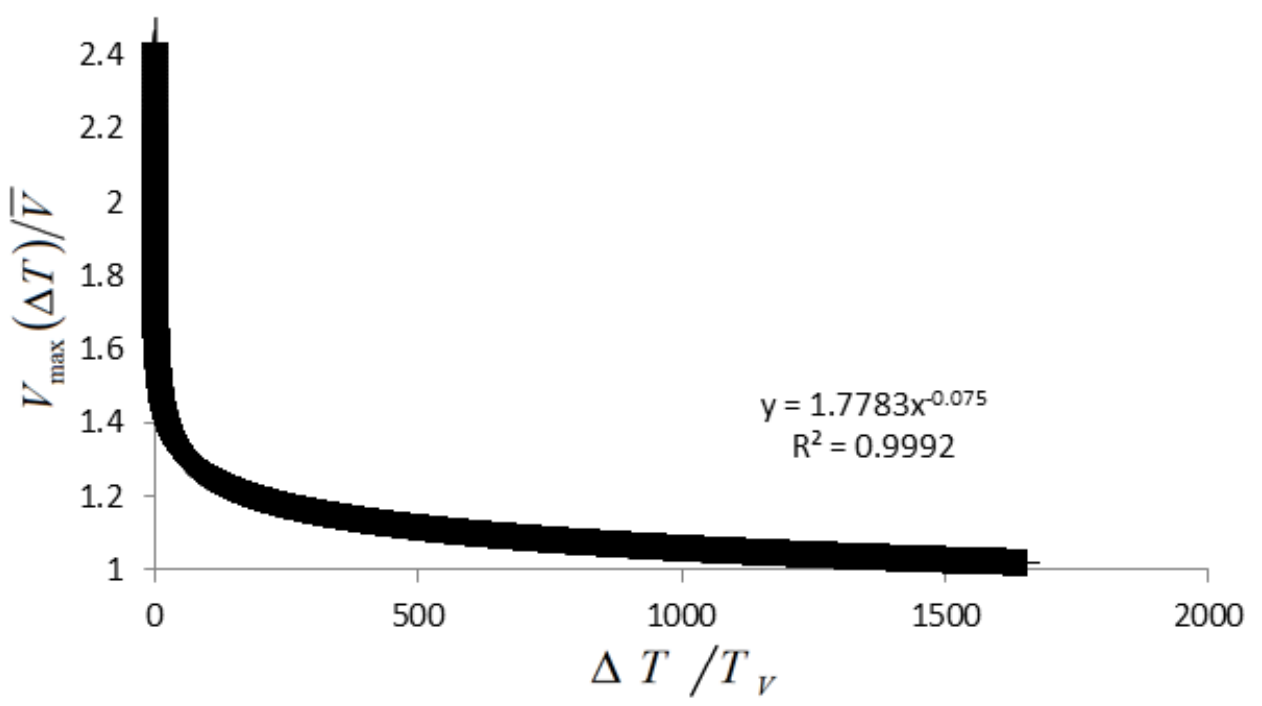

223

224

225

226

227

228

229

230 The fourth assumption is: "The ratio $V_{\max }(\Delta T) / \bar{V}$ depends on the fluctuation intensity $I$ (Eq.

Fig 2. The ratio $V_{\max }(\Delta T) / \bar{V}$ is plotted versus the ratio $\Delta T / T_{V}$ for the same dataset as in Fig. 1 .

The third assumption is: "When the time interval $\Delta \tau$ increases, the wind speed $V_{\max }(\Delta \tau)$ approximates zero, while it should tend to $\bar{V}$." This is illustrated in Fig. 3.

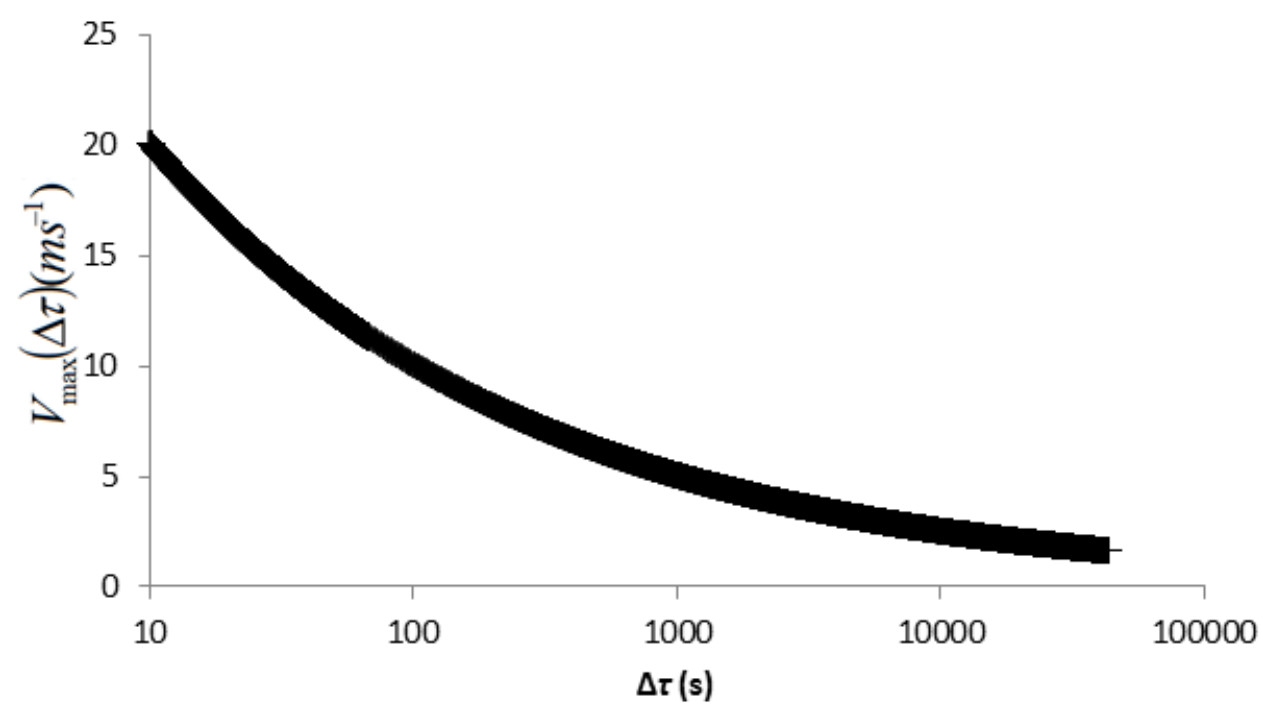

Fig 3 The maximum time-averaged wind speed $V_{\max }(\Delta \tau)$ is plotted versus the time interval $\Delta \tau$ for the same dataset as in Fig. 1. 4a)." In order to substantiate this assumption based on the data used in this study, the entire 232 set of experimental wind speed time series of the wind-tunnel boundary-layer flow case BL32330 were analyzed. The ratio $V_{\max }(\Delta T) / \bar{V}$ is plotted against the fluctuation intensity $I$ in Fig. 4, 234 clearly illustrating a strong linear relationship $\left(R^{2}=0.947\right)$. 


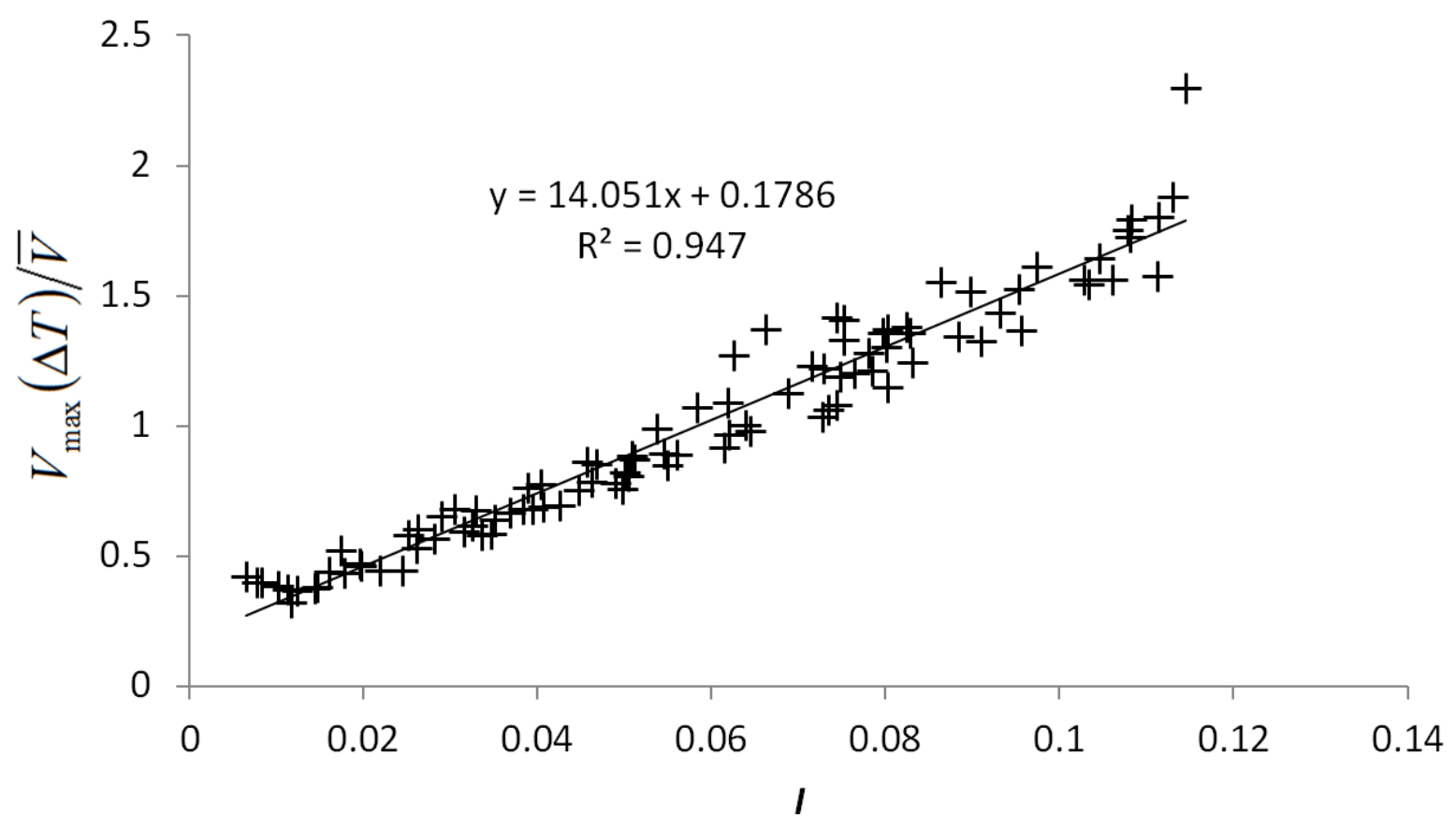

235

236

237

238

239

240

241

242

$243 \quad \frac{V_{\max }(\Delta \tau)}{\bar{V}}-1=b I\left(\frac{\Delta \tau}{T_{V}}\right)^{-0.3}$

244 The form of Eq. 8 is equivalent to the linear equation $y=b x$ where:

$245 \quad y=\frac{V_{\max }(\Delta \tau)}{\bar{V}}-1$

$246 \quad x=I\left(\frac{\Delta \tau}{T_{V}}\right)^{-0.3}$

247 If Eq. 5 is valid for wind speed then $y$ should clearly correlate with $x . y$ and $x$ are plotted in 248 Fig. 5. As for Fig. 4, the data points in Fig. 5 are from all the horizontal wind speed time 249 series of the laboratory experiment (BL3-0 case). The correlation coefficient $R^{2}$ is 0.87 , 250 which indicates that there is a significant linear relation. Furthermore it is noteworthy that the 251 parameter $b$ takes a value of 1.85 which is close to the indicative value of 1.5 that had earlier 252 been determined for concentration data. 


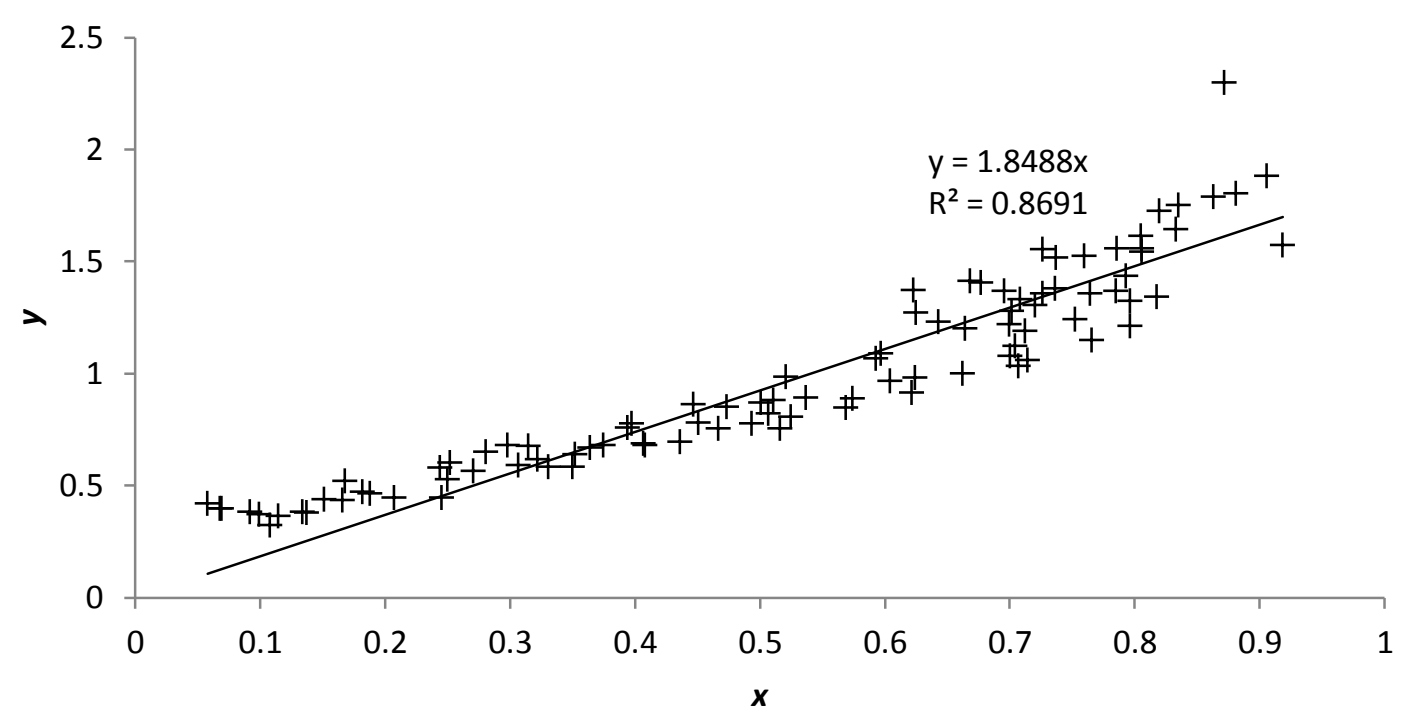

Fig 5 Examination of the validity of Eq. 5 for wind speed data based on all horizontal wind speed time series available from the rough-wall boundary-layer flow experiment in the wind tunnel.

However, since we are interested in the p.d.f.'s upper bound we go beyond the indicative maximum values measured in the experiments and instead focus on the extreme value $V_{\max }(\Delta \tau)$ reached within a time interval of infinite duration. It is noted that naturally such an extreme value cannot be verified experimentally. When comparing the measured peak value $V_{\max }^{\text {meas }}(\Delta \tau)$ with the expected extreme value $V_{\max }(\Delta \tau)$ for a specific sensor location, in theory the relation $V_{\max }^{\text {meas }}(\Delta \tau) \leq V_{\max }(\Delta \tau)$ will always hold true due to the ultimately finite length of the measured signal.

It is proposed that the extreme value $V_{\max }(\Delta \tau)$ can be approximated based on Eq. (5) given the values of the parameters $b$ and $v$. The present strategy is to fix the value of $v=0.3$ (the indicative value from Bartzis et al., 2008) and allow the $b$ parameter to be estimated from suitable calibrating data. This reduces the problem of estimating $V_{\max }(\Delta \tau)$ to estimating a single parameter, $b$.

Bartzis et al. (2008) have presented a method to estimate the parameter $v$, where $\left(V_{\max }(\Delta \tau) / \bar{V}\right.$ ) - 1 is plotted versus $\Delta \tau$. $V_{\max }(\Delta \tau)$ is the experimental maximum time-averaged wind speed which changes at every $\Delta \tau$. In other words at every $\Delta \tau$ a new wind speed time series is constructed and the maximum wind speed is calculated. For the following test $\Delta \tau$ ranges from 0.01 to $45000 \mathrm{~s}$. $V_{\text {mean }}$ is the experimental mean wind speed for $\Delta \tau$ equal to the measured time interval i.e. the original time series that is measured by the instrument. For the following test $\Delta \tau=0.01 \mathrm{~s}$. For this test we selected a random time series from the BL3-0 wind-tunnel experiment. Results are presented in Fig. 6. The equation $y=\alpha^{*}\left(x^{-\beta}\right)$ was fitted to the data, where $y$ is $\left(V_{\max }(\Delta \tau) / \bar{V}\right)-1$ and $x$ is $\Delta \tau$. In our case, the parameter $\beta$ is the desired $v$ parameter. For this dataset $\beta$ was found to be equal to 0.403 , which is very close to the previously determined value of 0.3 . Uncertainties arising after fixing the parameter $v$ are reflected in the value of $b$ for each particular location. 


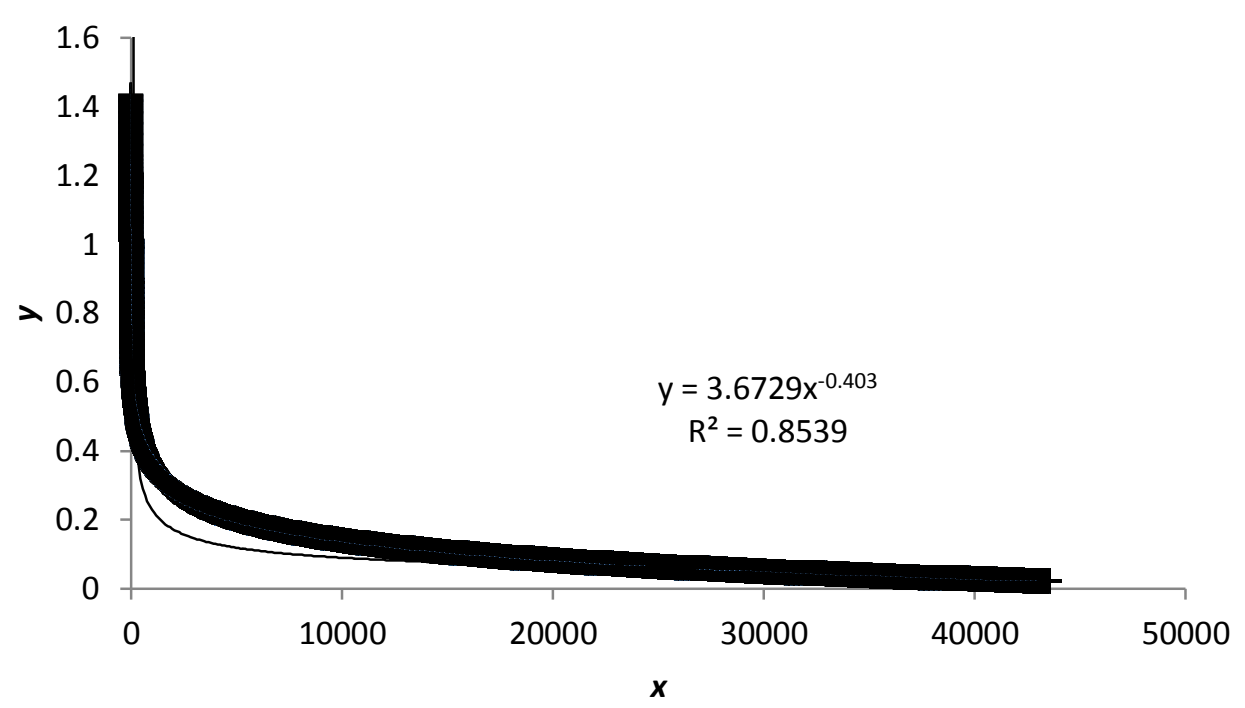

280

281

Fig $6\left(V_{\max }(\Delta \tau) / \bar{V}\right)-1$ is plotted versus the time interval $\Delta \tau$. The results correspond to a random wind speed time series of the BL3-0 case.

It is noted that for the parameters $b$ and $b^{\text {meas }}$ obtained from Eq. (5) using $V_{\max }(\Delta \tau)$ and $V_{\max }^{\text {meas }}(\Delta \tau)$ respectively, in theory $b^{\text {meas }} \leq b$ always holds true since $V_{\max }^{\text {meas }}(\Delta \tau) \leq V_{\max }(\Delta \tau)$.

With this approach we implicitly hypothesize the existence of a single value of $b$ that can qualify as the upper bound of all $b^{\text {meas }}$ values obtained at any location in the ASL flow at which the corresponding value of $V_{\max }^{\text {meas }}(\Delta \tau)$ is detected.

To close the model, it remains to estimate the value of the parameter $b$ in Eq. 5 .

In summary there are two groups of equations.

(1) The equations for the construction of the Beta distribution (Eqs. 2a, 2b, 3a, 3b and $3 c)$.

(2) The equation for the estimation of the maximum time-averaged wind speed (Eq. 5).

The equations of the first group use the equation of the second group through the parameter $\eta$ (Eq. 3c).

\subsection{Application of the method}

The methodology used in this study includes the following steps:

1. Estimation of the parameter $b$ from an analysis of experimental and numerical datasets. In our study we use near-surface turbulent flow signals that are available from a dense sensor network, offering sufficient coverage of a diverse set of ASL flow scenarios, also including data measured within and above urban environments. The wind speed time series should be of high time resolution, statistically stationary and have a sufficiently long duration to ensure that relevant statistics are derived with high levels of statistical confidence. 
2. Calculation of the mean wind speed, variance and time scale parameter $T_{V}$ from each measured wind speed time series from the calibrating data.

3. Identification of the peak value $V_{\max }^{\text {meas }}(\Delta \tau)$ and estimation of the corresponding local parameter $b^{\text {meas }}$ from Eq. (5) for each wind speed time series. In this study, the selected time interval $\Delta \tau$ is equal to the time resolution of the experimental/simulation data.

4. Estimation of the parameters for the wind speed Beta p.d.f. as described by Eqs. (2a, b) and ( $3 \mathrm{a}, \mathrm{b}, \mathrm{c}$ ) for each location. The values of the required input variables $\bar{V}, \sigma_{V}^{2}$ and $T_{V}$ are derived from the reference datasets (see point 2 . above). The single missing parameter is the coefficient $b$.

\section{Derivation of a suitable value for $b$.}

6. Evaluation of the accuracy and robustness of the single value for $b$ derived from the calibrating data by testing the model based on independent wind speed measurements from field experiments.

It is emphasized that we look for a p.d.f. that produces a relatively simple but adequate approximation. Thus, the present methodology is based on the assumption that if a theoretical c.d.f. can reproduce the real threshold wind speeds for various probabilities then the p.d.f. used is considered a good approximation.

\section{Data and Test Cases}

For the estimation of the parameter $b$ and the construction of the statistical model we use data from wind-tunnel experiments and numerical simulations. The performance of the statistical model is evaluated in Sect. 5 based on hourly wind speed measurements taken at various field sites.

\subsection{Wind-tunnel experiments}

The first two flow datasets analyzed in this study to derive the model coefficient $b$ stem from boundary-layer wind-tunnel measurements conducted at the Environmental Wind Tunnel Laboratory (EWTL) of the University of Hamburg. The datasets are part of the CEDVALLES reference database that offers time-averaged statistics as well as time-resolved data for different types of boundary-layer flow and dispersion scenarios under neutral stability conditions. The validation datasets are freely available and described in detail in Fischer et al. (2010).

CEDVAL-LES contains data for various levels of geometric complexity and surface roughness characteristics. In the database, the term "complexity" refers to the configuration of the flow scenario covered in the experiment, ranging from complexity 0 denoting simple rough-wall boundary-layer flows, over flows around isolated obstacles or within obstacle arrays (complexities 1 or 2 , respectively) to flows in semi-idealized urban environments (complexity 3) or realistic city layouts (complexity 4). Two of the available cases were selected for this study: (1) boundary-layer flow over a very rough surface (CEDVAL-LES 
complexity 0; case reference: BL3-0) and (2) urban flow within and above a semi-idealized city geometry (complexity 3; "Michel-Stadt" case reference: BL3-3). In both cases the flow was physically modelled under a scale of 1:225 and point-wise velocity measurements were conducted by means of 2D laser Doppler anemometry (LDA). The setup of the very rough boundary-layer flow (BL3-0) was used as the inflow boundary layer for the semi-idealized city case (BL3-3).

\subsubsection{Boundary layer over very rough surface}

With a power-law profile exponent of $\alpha=0.27$ and a roughness length of $z_{0}=1.53 \mathrm{~m}$, the boundary-layer flow (complexity 0 case) shows roughness characteristics of flow above an urban environment. In this case, the buildings are not directly represented, but their aerodynamic effect on the approach flow boundary layer is physically modelled by means of floor-roughness elements.

At sufficient distance from the tunnel inlet and from the floor-roughness elements, the boundary layer was verified to be horizontally homogeneous. Measurements are only taken above the blending height, where only the integrated effect of the surface roughness is represented in the flow characteristics. The 2D-LDA was operated consecutively in two measuring modes to acquire two components of the velocity vector at a time: the streamwise $(U)$ and spanwise $(V)$ velocities $(U V$-mode) and the streamwise and vertical $(W)$ velocities $(U W$-mode). Data are available in terms of vertical profiles and horizontal transects perpendicular to the mean inflow direction. All velocity data were scaled to a full-scale reference height of $z_{\text {ref }}=100 \mathrm{~m}$ (444.44 mm model scale) with reference wind speeds $U_{\text {ref }}$ ranging between 4.75 and $6 \mathrm{~m} \mathrm{~s}^{-1}$. With a measurement duration of $3 \mathrm{~min}$ (corresponding to $11 \mathrm{~h}$ full scale) per locations, the derived velocity statistics offered a high level of statistical representativeness.

For the present study, wind speed time series with a full-scale resolution of $\Delta \tau=0.01 \mathrm{~s}$ were analyzed at 96 points for $U V$ measurements. It should be noted that all time-series were resampled to the same time resolution.

\subsubsection{Flow in a semi-idealized city}

The semi-idealized urban geometry includes typical features of Northern and Central European cities like courtyards, oblique road arrangements, squares and complex intersections. Three building heights are included in the model: 15, 18 and $24 \mathrm{~m}$ full-scale (see. Fig. 7 top left). All buildings had flat roofs.

Velocity measurements were conducted with the LDA in $U V$-mode, providing information about the horizontal winds within and above the city. The streamwise reference velocity at a height of $100 \mathrm{~m}$ was kept at $6 \mathrm{~m} / \mathrm{s}$ and was monitored during each measurement run. The reference velocity was verified to be sufficiently high to guarantee Reynolds number independence of derived flow statistics within and above the urban canopy. Three groups of wind speed measurements were used in this study: (1) 40 vertical profiles distributed at various points throughout the city (Fig. 7, bottom left), (2) detailed measurements on dense 
horizontal grids at heights of 2, 9 and $18 \mathrm{~m}$ full-scale within the main city area (340 $\mathrm{m} \times 340$ m), containing 383 measurement points per level (Fig. 7, top right); (3) measurements on a coarse horizontal grid above the city centre at full-scale heights of 27.5 and $30.2 \mathrm{~m}$, containing 252 data points at each height (Fig. 7, bottom right).

The total number of signals analyzed is 2,158. Again the time series were resampled to a resolution of $0.01 \mathrm{~s}$.
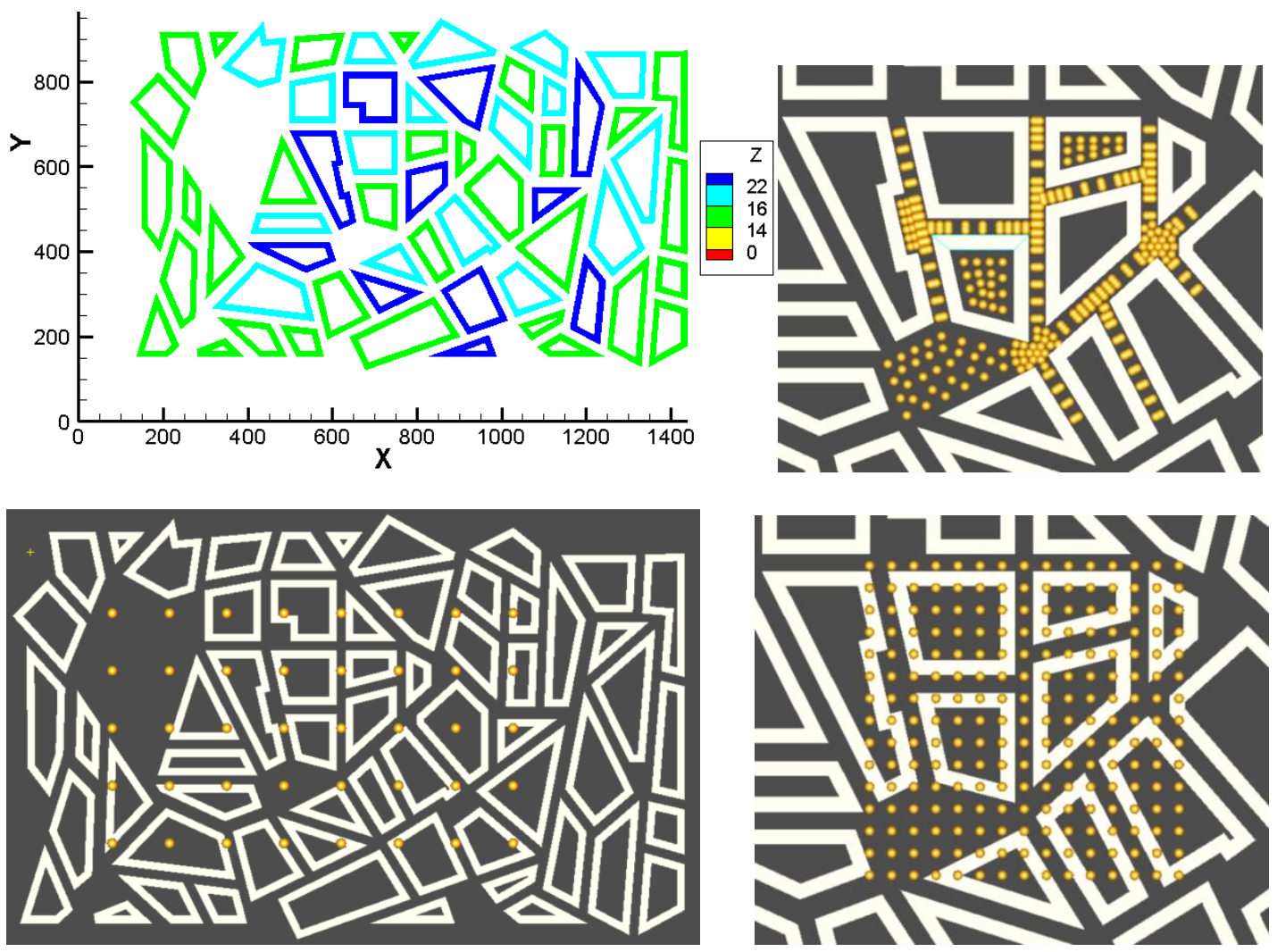

Fig 7 Top left: the idealized city domain (dimensions given in full scale, dimensions $X, Y$ and $Z$ are in meters); top right: densely-spaced measurement locations within the urban canopy layer at elevations of $2 \mathrm{~m}, 9 \mathrm{~m}$ and 18 $\mathrm{m}$; bottom left: vertical profile locations; bottom right: densely-spaced measurement locations above roof top at heights of $27 \mathrm{~m}$ and $30 \mathrm{~m}$.

\subsection{Direct numerical simulations}

The second type of data analyzed in this study stems from simulations of urban flow fields in an idealized urban roughness generated by direct numerical simulation (DNS). With the DNS approach, turbulence is directly resolved down to the small dissipative eddy scales. In order to facilitate this computationally, the flow is simulated at lower Reynolds numbers compared to the ones typically encountered in the atmospheric boundary layer. The Reynolds numbers that can be realized, however, are comparable to those typically achieved in boundary-layer wind-tunnel experiments as those described in the preceding sections. Compared to other turbulence-resolving CFD approaches like for example large-eddy simulation (LES), the accuracy of flow simulations with DNS is not affected by errors resulting from turbulence modelling. Hence, DNS data, after appropriate accuracy checks, can be used as reference data similar to experimental measurements. The significant computational requirements involved 
403

404

405

406

407

408

409

410

411

412

413

414

415

416

417

418

419

420

421

422

423

424

425

426

in performing DNS, however, currently restrict the applicability of the technique to simple flow scenarios at low Reynolds numbers.

The DNS code that had been used to generate the data analyzed in this study is the research code CgLES developed at the University of Southampton specifically for performing massively-parallel DNS and LES computations. The code is parallelized with the Message Passing Interface (MPI) and a flexible multi-block mapping strategy is used to deal with complex geometries. The Navier-Stokes equations are discretized using second-order central finite differences in space and a second-order Adams-Bashforth scheme in time based on the pressure correction method. The Poisson equation for pressure is solved using a multigrid method. A detailed description of the numerical techniques involved in the DNS as well as examples of previous studies with the code can be found, e.g., in Yao et al. (2001), Branford et al. (2011) or Coceal et al. (2006, 2007, 2014).

Flow simulations were conducted in a geometry comprised of 64 cubical obstacles of height $H$ that were set up in a regular array consisting of 8 rows of 8 obstacles. The computational domain was of size $16 H \times 16 H \times 8 H$. Periodic boundary conditions were prescribed in horizontal directions, free-slip conditions at the upper domain boundary and no-slip and impermeability conditions at the bottom of the domain and at solid surfaces. The flow was driven by a constant body force that resulted in a roughness Reynolds number of $\operatorname{Re}_{\tau}=u_{\tau} H / v$ $=500$, where $u_{\tau}$ is the total wall friction velocity and $v$ is the kinematic viscosity. Sensitivity studies for this setup (Coceal et al. 2006, 2007) demonstrated that the selected resolution of the uniform grid of $H / 32$ is sufficient to adequately resolve the flow down to the dissipative scales. Data is available for two wind directions $\left(0^{\circ}\right.$ and $\left.45^{\circ}\right)$ under neutral stratification. Fig. 8 shows snapshots of the instantaneous horizontal wind speed magnitudes at a height of $Z=$ $0.5 \mathrm{H}$ for both wind directions, illustrating the complexity of the canopy layer flow field.

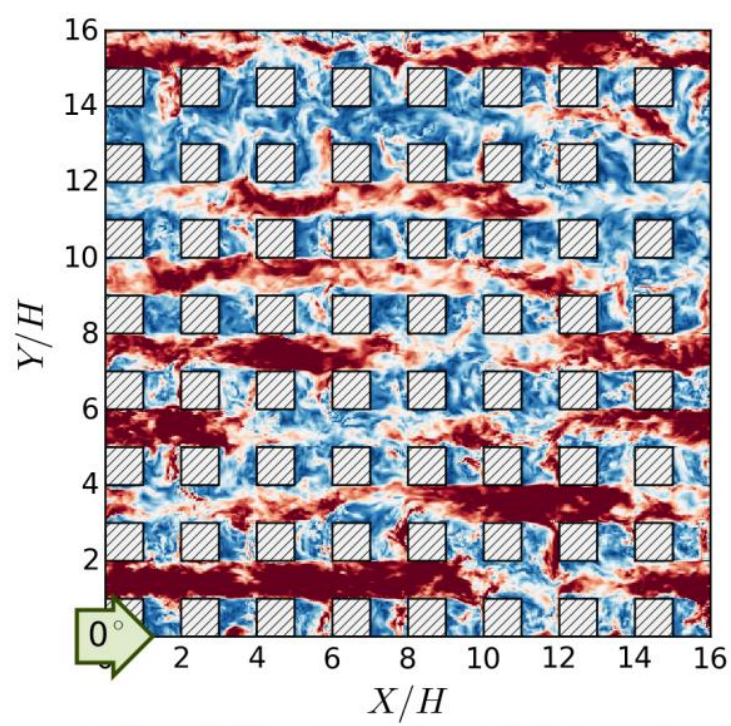

$\begin{array}{lllllllllll}0.0 & 0.5 & 1.0 & 1.5 & 2.0 & 2.5 & 3.0 & 3.5 & 4.0 & 4.5 & 5.0\end{array}$

427

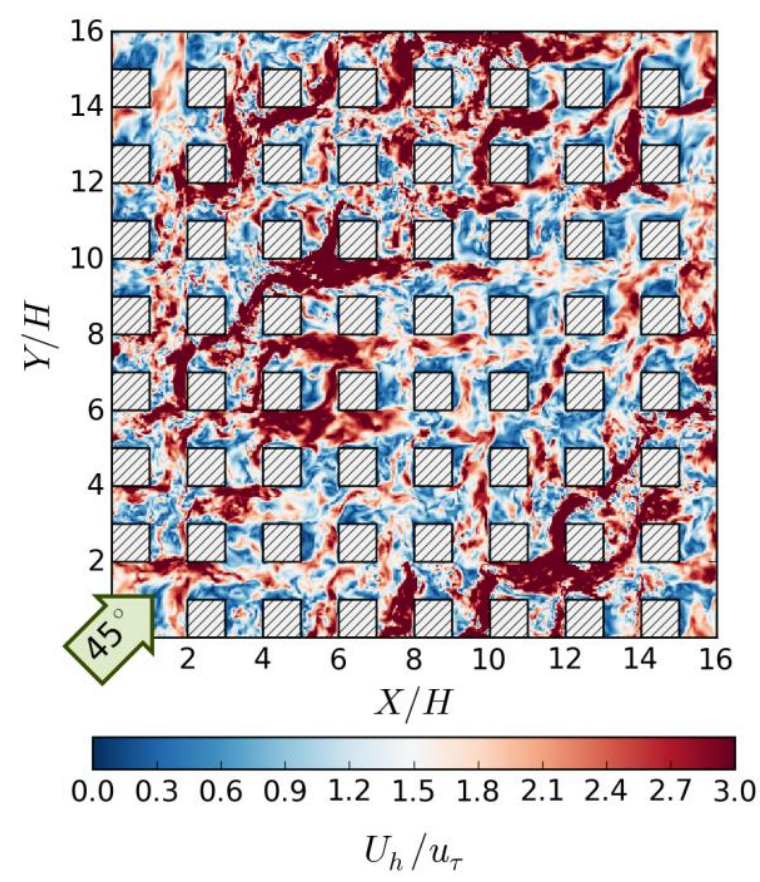


Fig 8 Plan views of the computational domain of the DNS including contours of instantaneous snapshots of the non-dimensional horizontal velocity magnitude, $U_{h} / u_{\tau}$, at half the building height $(Z=0.5 H)$. Left: $0^{\circ}$ forcing direction; right: $45^{\circ}$ forcing direction.

Wind speed time series at 896 locations overall (for both wind directions) were analyzed in this study, each of them having a length of $140 T$, where $T$ is the eddy turnover time defined as $T=H / u_{\tau}$. As done with the wind-tunnel data, the DNS time series were resampled to a non-dimensional time resolution of 0.01 . The data locations are distributed in two horizontal planes covering the entire simulation domain at two different heights: $Z=0.5 H$ and $Z=1.5 H$. Time series were extracted in the centre of each street and intersection. The DNS computation were performed on a supercomputer and required a total spin-up time of 12 days on 124 nodes for both the $0^{\circ}$ and $45^{\circ}$ runs, after which the simulations ran for 10 days $\left(0^{\circ}\right.$ case) and 13 days ( $45^{\circ}$ case) on 248 nodes to collect flow time series and statistics.

\section{Estimation of the $b$ parameter}

\subsection{Wind-tunnel flow fields}

\subsubsection{Boundary-layer flow}

The autocorrelation time $T_{V}$ is calculated from the autocorrelation function $R_{\nu}(\tau)$ on the interval from 1.0 to 0 . The wind speed time series are considered to be characterized by a sufficiently high temporal resolution.

At each sensor location, an experimental peak $V_{\max }^{\text {meas }}(\Delta \tau)$ is identified and a $b^{\text {meas }}$ value is estimated from Eq. 5 using $V_{\max }^{\text {meas }}(\Delta \tau)$. The values of $b^{\text {meas }}$ range from 1.5 to 7.2. The value of 5 , however, is only exceeded three times out of 96 (i.e. 3.1\%) and seems to point more to 'outliers' behavior.

It is evident that if the proposed model is valid and a single value for $b$ exists this value has to be greater than 5 . This dataset and the two other datasets (discussed in detail in the following Sects. 4.1.2. and 4.2) clearly indicate that a value of $b=6$ is appropriate for these flow scenarios.

For a randomly selected sensor of the BL3-0 case, the Beta and the experimental p.d.f.s and c.d.f.s are plotted in Fig. 9 (first row). The agreement between the model curve and the experimental data points is very good.
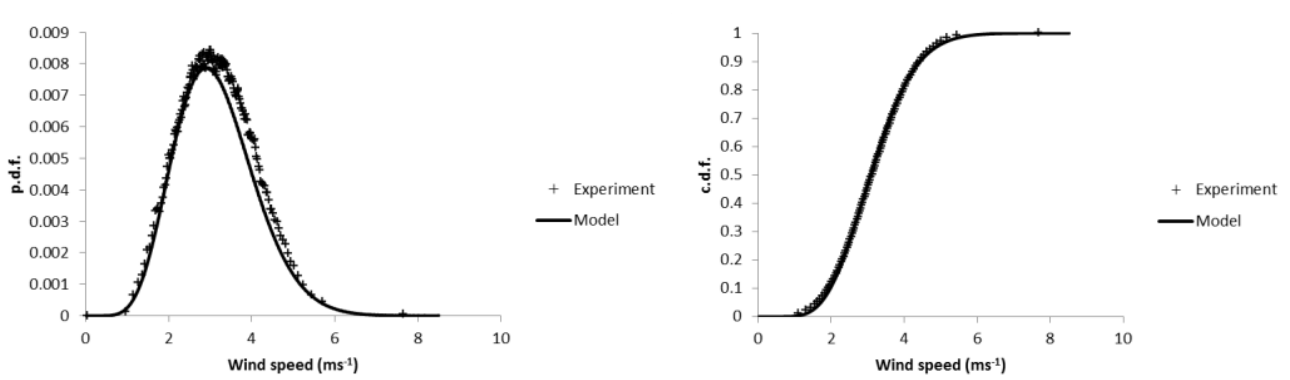

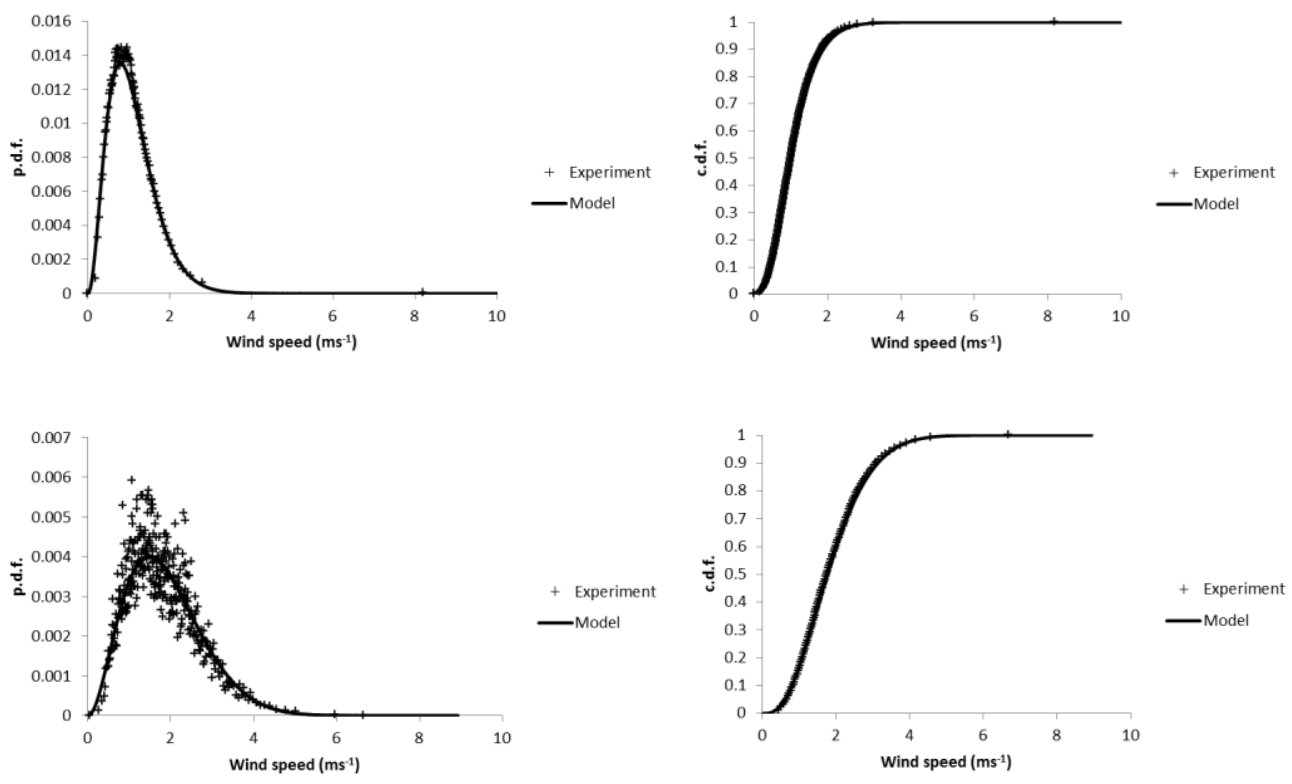

460

461

462

463

464

465

466

467

468

469

470

471

472

473

474

475

476

477

478

479

480

481

482

Fig 9 Experimental and theoretical p.d.f.s and c.d.f.s using the Beta distribution for a randomly selected sensor of the BL3-0 case (first row); for a randomly selected sensor of the BL3-3 case (second row) and for a randomly selected sensor of the DNS experiment (third row). The parameter $b$ is set to 6 .

\subsubsection{Semi-idealized city}

As a next step, the robustness of using a value for $b$ equal to 6 was examined by analyzing flow data acquired in the semi-idealized city wind-tunnel model.

As for the boundary-layer flow data, the $b^{\text {meas }}$ is obtained based on the analysis of all 2,158 signals. The $b^{\text {meas }}$ ranges from 0.63 to 7.1 . Again, only at three of the available measurement positions a $b^{\text {meas }}$ value larger than 5 was obtained. The proposed value of $b$ (equal to 6 ) is also relatively close to the maximum value of 7.1.

As for the boundary layer flow case analyzed above, the agreement between the Beta and the experimental p.d.f.s and c.d.f.s for an example time series from a randomly selected sensor location is very high (Fig. 9, second row).

\subsection{Direct numerical simulations}

The analysis of the wind-tunnel data clearly supports the proposed methodological approach. In a next step, the DNS data of flow in an idealized urban roughness is analyzed in a similar manner.

As with the experimental data, the DNS dataset is first analyzed with regard to determining an appropriate value of the $b$ parameter. For each sensor, a peak $V_{\max }^{\text {meas }}(\Delta \tau)$ is identified from the simulation data and the corresponding $b^{\text {meas }}$ value is estimated from Eq. (5). The $b^{\text {meas }}$ range is from 1.1 to 5.56. Only 10 values are larger than 5 and the proposed $b$ value (equal to 6 ) is relatively close to the maximum value 5.56 . 
The Beta and the experimental p.d.f.s and c.d.f.s from time series at a randomly selected point of the DNS flow simulation is presented in Fig. 9 (third row) and again reveal a high level of agreements between the statistical model and the reference data.

It is worth highlighting that it is rather impressive that two completely different kinds of data sources, the wind-tunnel measurements and the DNS, corresponding to very different nearsurface flow scenarios show such a similar range of values determined for $b^{\text {meas }}$. This provides strong support for the hypothesis that a single representative maximum value of the $b$ parameter can be derived, which could then be applied to various types of ASL flows.

\section{Model evaluation based on field data}

Based on the analysis of the three different calibrating datasets presented above, an upper value of the parameter $b$ equal to 6 was derived. However, the flow scenarios analyzed to derive this model parameter represent quite idealized cases of ABL flow (e.g. with respect to the isothermal conditions/neutral stratification and stationarity of the flow). In order to demonstrate the applicability of the model to real-world ABL flow scenarios, in a next step the model is evaluated based on independent field measurements. These consist of hourlyaveraged in-situ wind-speed measurements from multiple ground-based sensors available over the course of several months, which reflect the true variability of the natural atmospheric boundary layer in terms of wind-speed trends (e.g. through the propagation of meso-scale systems) and stratification effects.

For this study, the Greek Public Power Corporation (https://www.dei.gr/en) provided hourly wind-speed data for the period from 1 January 2012 to 31 August 2012 from 7 meteorological stations (Vevi, Florina, Koilada, PPC village, Pentabrysos, Petrana and Pontokomi) located in the western part of Greece (see Fig. 10). All velocity sensors are located in urban areas, the measurement height is $10 \mathrm{~m}$ above ground and the averaging period of the signals is $1 \mathrm{~h}$.
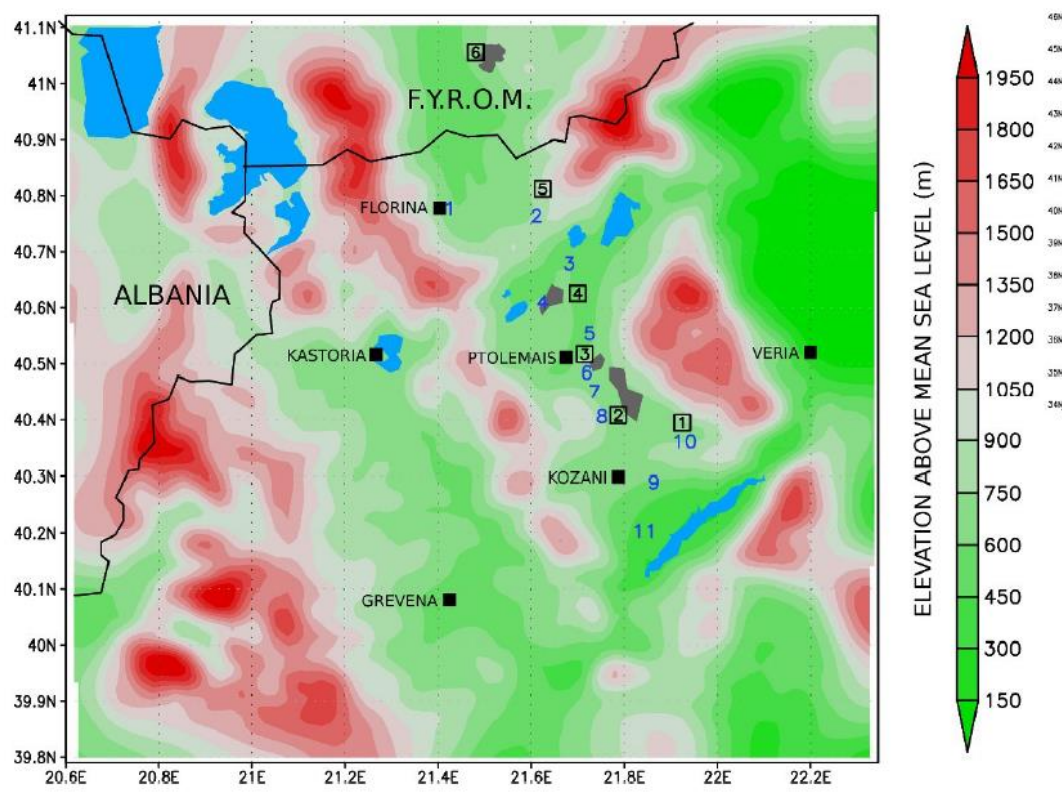

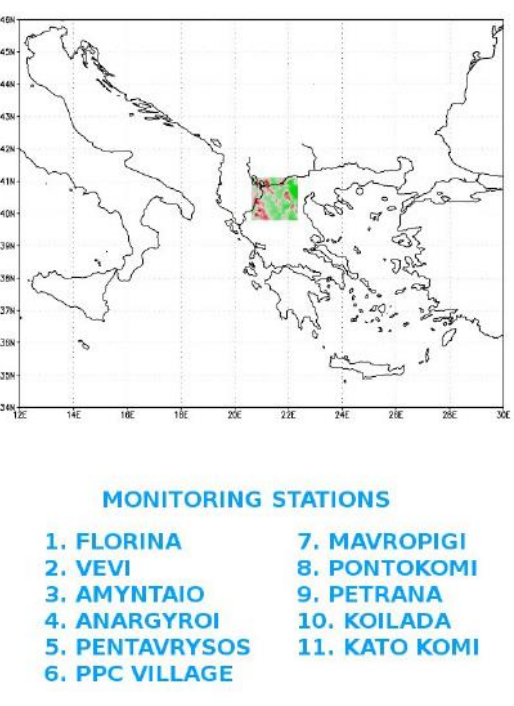


Fig 10 Topography of Western Macedonia, Greece, where the meteorological stations are located. The black numbered boxes indicate power plants, gray areas mines. The blue numbers indicate the location of meteorological measurement stations, with names listed on the right. Data available for this study are from the stations in Vevi, Florina, Koilada, PPC village, Pentabrysos, Petrana and Pontokomi.

Initially a comparison is performed in terms of the theoretical value of $V_{\max }(\Delta \tau)$ as derived from the Bartzis et al. (2008) model and the measured $V_{\max }^{\text {meas }}(\Delta \tau)$ at all stations (see Fig. 11). The model provides a success rate of $85.7 \%$ (only one value is below the $1: 1$ line), which supports the hypothesis that the proposed theoretical $V_{\max }(\Delta \tau)$ can serve as an upper bound of the corresponding measured $V_{\max }^{\text {meas }}(\Delta \tau)$.

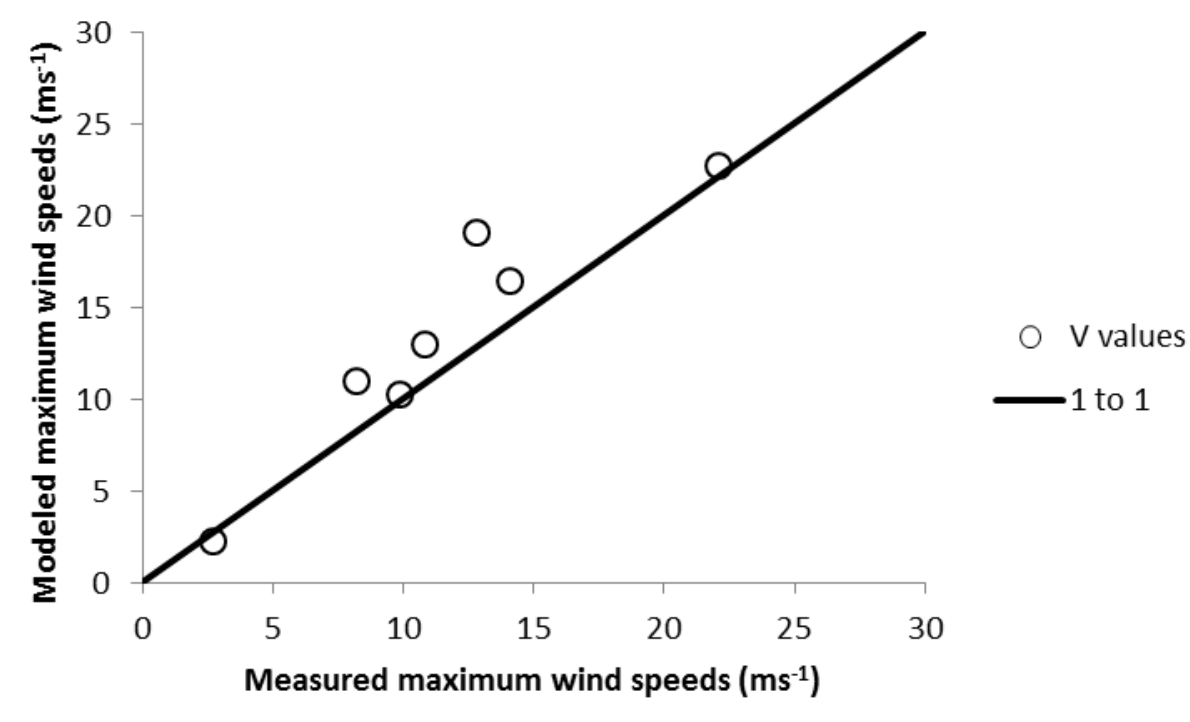

Fig 11 Modelled versus measured peak wind speeds at the 7 field measurement stations. The straight line indicated the 1:1 relationship.

In the following analysis the performance of the Weibull distribution is also tested. This distribution was fitted to the data and its parameters were calculated with the maximum likelihood estimation. The results of the $99 \%$ threshold (c.d.f. $(V)=0.99)$ are analyzed in order to test the performance of the statistical model at the upper tail of the distribution. Based on the analysis of the calibrating data discussed in the preceding section, the Beta distribution was configured with a $b$ value of 6 . In Fig. 12 scatter plots are presented comparing the calculated wind speed $V(\Delta \tau)$ from the Beta and Weibull distributions and the ones derived from the field data corresponding to cumulative probabilities of c.d.f. $(V)=0.25,0.50,0.75$ and 0.99 . The Beta distribution performs slightly better than the Weibull distribution in the higher wind-speed range for probabilities of $0.25,0.50$ and 0.99 . 
(a) c.d.f. $(V)=0.25$

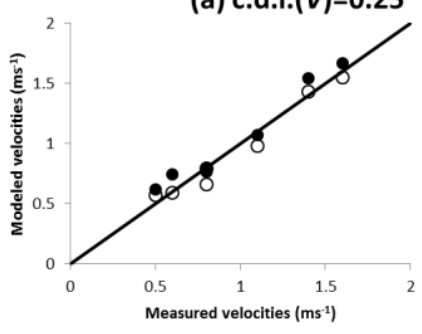

(c) c.d.f. $(V)=0.75$

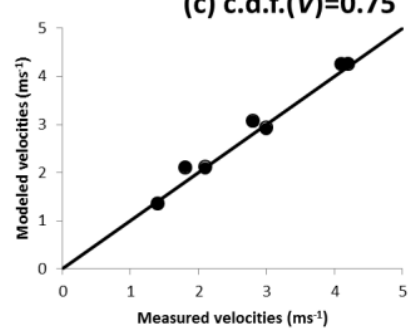

(b) c.d.f. $(V)=0.50$

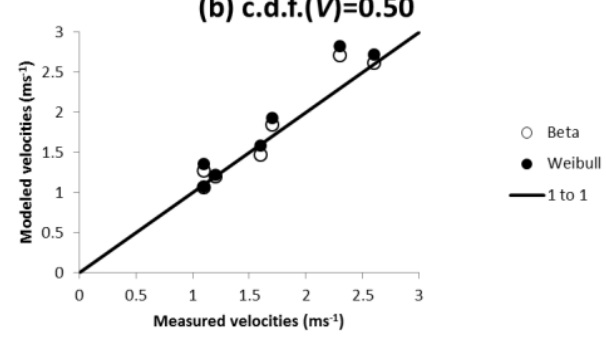

(d) c.d.f.(V)=0.99

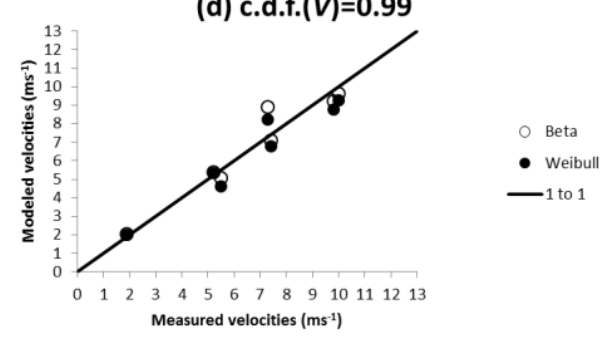

Beta

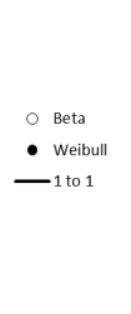

533 Fig 12 Modelled (Beta and Weibull) versus measured wind speeds from the field experiment corresponding to (a) c.d.f. $(V)=0.25$, (b) c.d.f. $(V)=0.50$, (c) c.d.f. $(V)=0.75$ and (d) c.d.f. $(V)=0.99$.

The remaining critical question is how individual c.d.f.s derived from the Beta distribution compare with the counterparts from the field measurements at each location. In Fig. 13 the measured and modelled c.d.f.s for percentiles between $75^{\text {th }}$ and $100^{\text {th }}$ are shown for three stations (Vevi, PPC village and Pontokomi). At these and the other locations not shown here, the model presents good agreement with the field data.

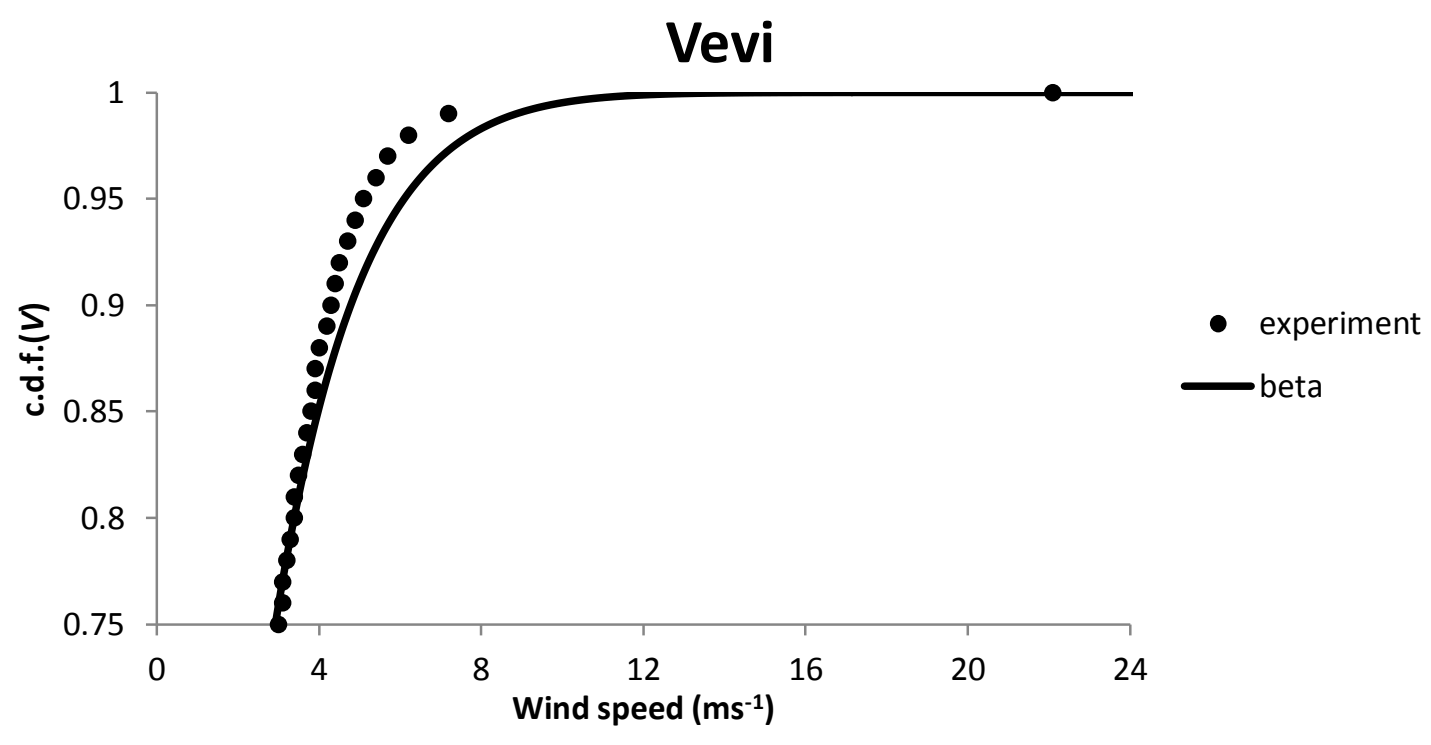



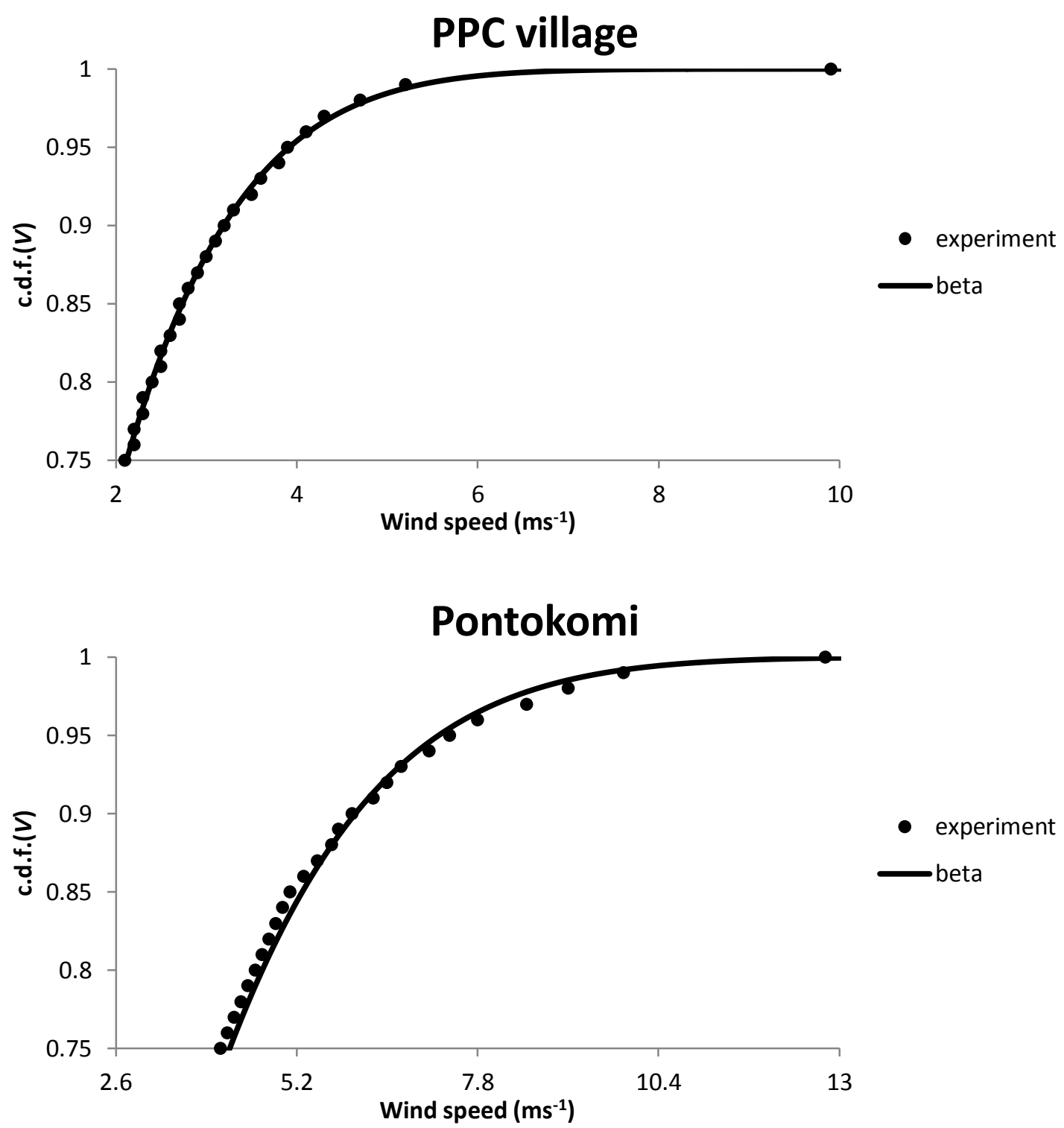

Fig 13 Modelled versus measured c.d.f. results for percentiles between the $75^{\text {th }}$ and $100^{\text {th }}$ for three meteorological stations of the Western Macedonia region of Greece.

Concerning the atmospheric stability it should be noted that the wind tunnel and the DNS experiments modelled neutral conditions. However the present field experiment covers all the stability conditions and the Beta distribution performs very well under all conditions. Using a value of $b=6$ when applying the model to independent datasets from field measurements shows that the value seems to be a good choice when dealing with ASL wind speeds.

\subsection{Effect of the time interval $\Delta \tau$ on the performance of the model}

A further testing of the universal nature of the proposed Beta model is conducted by repeating the above analysis for different $\Delta \tau$.

553 In Fig. 14 the peak time-averaged wind speeds based on Eq. 5 and the field experiment are 554 plotted for different $\Delta \tau$ for an example station. The horizontal axis is presented using a 555 logarithmic scale. For $\Delta \tau=1 \mathrm{~h}$ the model wind speed is higher than the experimental peak 
559

560

561

562

563

564

565

566

567

568

569 wind speed, as expected. For $\Delta \tau$ between $10 \mathrm{~h}$ and $100 \mathrm{~h}$ the model slightly underestimates the experimental values, while overestimating again after $100 \mathrm{~h}$. For $1000 \mathrm{~h}$ the $V_{\max }(\Delta \tau)$ of both model and experiment approximate the mean wind speed.

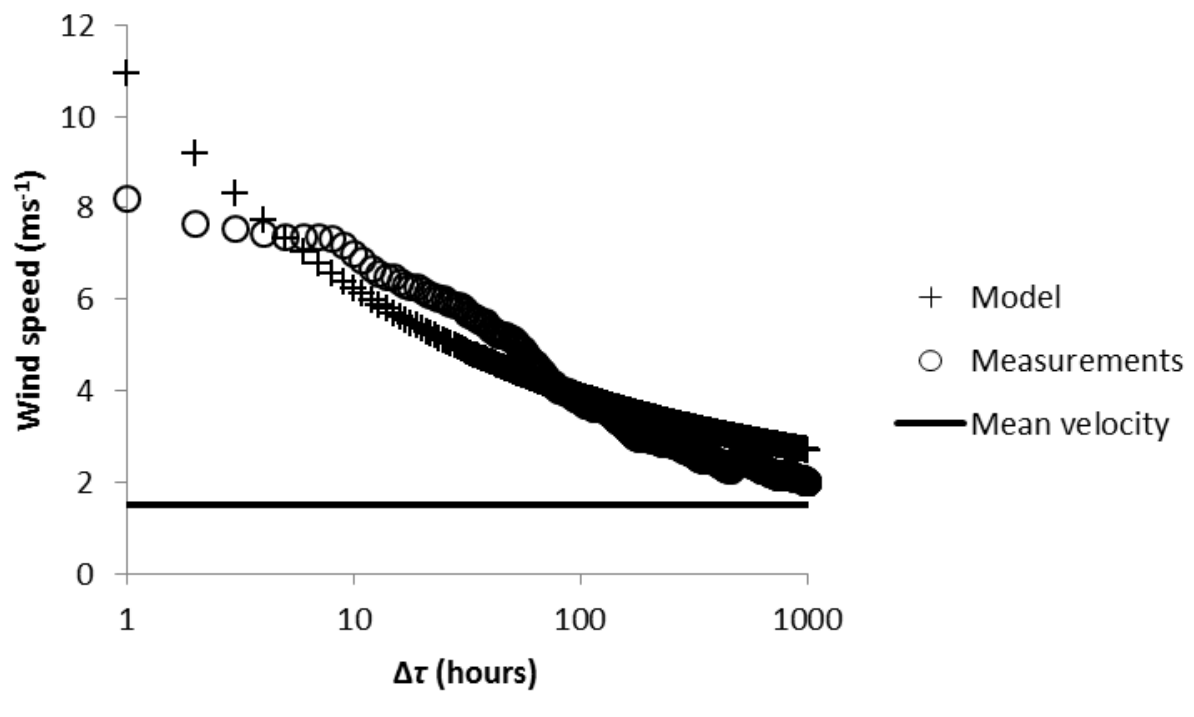

Fig. 14 Examination of Eq. 5 against field measurements for various $\Delta \tau$ for data from an example station.

In Fig. $15 V(\Delta \tau)$ obtained from the Beta distribution model and the values derived from one of the field stations are presented. $\Delta \tau$ ranges from $1 \mathrm{~h}$ to $24 \mathrm{~h}$. The results correspond to cumulative probabilities of c.d.f. $(V)=0.25,0.50,0.75$ and 0.99 . For c.d.f. $(V)=0.25$ and 0.5 the wind speed increases with $\Delta \tau$ while for 0.75 and 0.99 it decreases with $\Delta \tau$. This indicates that with the increase of $\Delta \tau$ the distribution becomes more leptokurtic. The model shows the same tendency as the experiment. For the specific station the largest deviation of the model from the experiment is observed for c.d.f. $(V)=0.25$ and for $\Delta \tau=24 \mathrm{~h}$. On the other hand the best performance of the model is observed for c.d.f. $(V)=0.5$ over the entire range of $\Delta \tau$.

(a) c.d.f. $(V)=0.25$

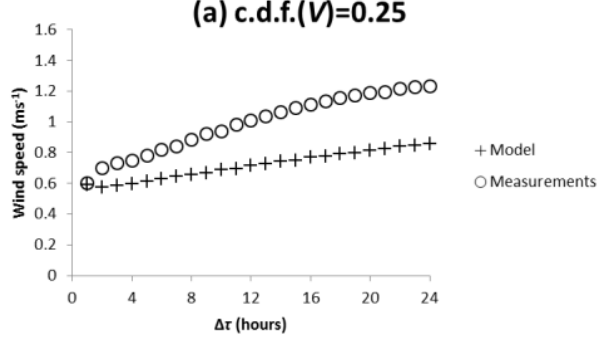

(c) c.d.f. $(V)=0.75$

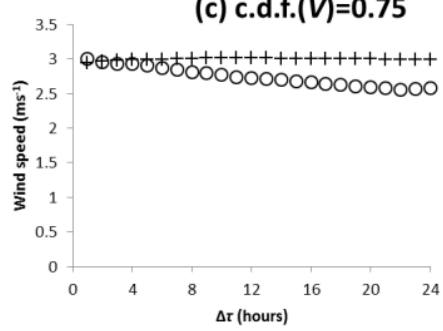

(b) c.d.f. $(V)=0.5$

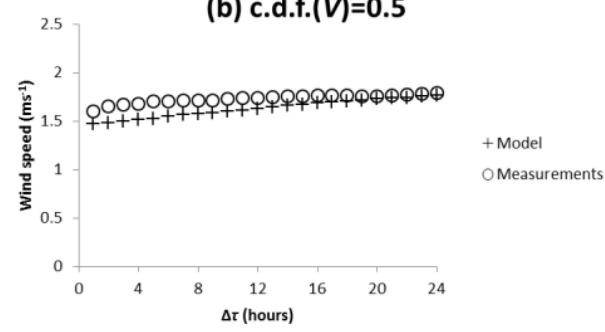

(d) c.d.f.(V)=0.99

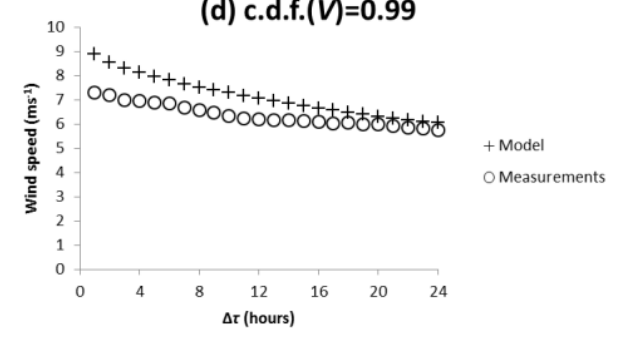

Fig 15 Beta distribution versus measured wind speeds for an example station corresponding to (a) c.d.f. $(V)=$ 0.25 , (b) c.d.f. $(V)=0.50$, (c) c.d.f. $(V)=0.75$ and (d) c.d.f. $(V)=0.99$. 
574 By using the properties of the Beta p.d.f. in combination with a model for estimating extreme

575 values based on readily available turbulence statistics (Bartzis et al., 2008), this study 576 demonstrated that this novel modelling approach can reliably predict the upper margins of 577 wind speeds encountered in the ASL.

578 The problem itself is quite complex and adequate validation studies require extensive 579 experimental datasets. Such comprehensive validation efforts exceed the scope of a single 580 publication, but the work presented here represents a significant first step towards a thorough 581 testing of the proposed methodology.

582 The selected calibrating data for constructing the proposed model are representative of 583 different scenarios of turbulent wind flow in the ASL: a rough boundary layer without 584 buildings, a typical European urban micro-environment and an urban-like arrangement of 585 cubical buildings. The sensor locations cover the boundary layer and the urban canopy-layer 586 characteristics. The temporal resolution of the wind-speed signals covers a wide range of 587 fluctuation intensities.

588 The performance of the model was successfully evaluated based on long-term independent 589 field measurements (hourly averages), which cover the true variability of ABL flows in terms 590 of wind-speed trends through the propagation of meso-scale systems and stratification effects. 591 Concerning the atmospheric stability it should be noticed that the wind tunnel and the DNS 592 experiments were conducted under neutral conditions. The field experiment, however, 593 covered a wide range of stability conditions that can be encountered in natural ASLs and the 594 Beta distribution performed very well under all conditions.

595 From the results obtained the following main conclusions are drawn:

596 1. The approximation of the statistical behavior of the abovementioned wind speed variability 597 with a Beta distribution p.d.f. was shown to be satisfactory.

598 2. The important issue of the extreme value in the Beta distribution is properly addressed by 599 an adaptation of the Bartzis et al. (2008) model.

600 3. The present work proposes $b=6$ and $v=0.3$ in Eq. 5.

601 4. With an increase of the averaging time interval the wind-speed distributions of the model 602 and experiment become more leptokurtic.

603 The new model can broaden the capability of ensemble-averaged computational models such 604 as Reynolds Averaged Navier Stokes-CFD models to estimate the wind-speed p.d.f. provided 605 that reliable predictions of mean wind speeds, wind-speed fluctuations and integral time 606 scales are available from these computations. 
The authors would like to thank Bernd Leitl of the Environmental Wind Tunnel Laboratory at the University of Hamburg for providing access to the reference database (CEDVAL-LES; https://www.mi.zmaw.de/index.php?id=6339). The authors would like to thank the Greek Public Power Corporation for providing access to the field measurements.

\section{References}

Aidan J (2011) Wind Speed distribution and Performance of some selected wind turbines in Jos, Nigeria. Latin Am J Phys Educat 5:457-460

Bartzis JG, Efthimiou GC (2010) Maximum individual exposure estimation using CFD RANS modelling. 13th conference on harmonisation within atmospheric dispersion modelling for regulatory purposes, 1-4 June, 2010, Paris, France.

Bartzis JG, Efthimiou GC, Andronopoulos S (2015) Modelling short term individual exposure from airborne hazardous releases in urban environments. J Hazard Mater 300:182-188

Bartzis JG, Sfetsos A, Andronopoulos S (2008) On the individual exposure from airborne hazardous releases: the effect of atmospheric turbulence. J Hazard Mater 150:76-82

Beljaars ACM (1987) The influence of sampling and filtering on measured wind gusts. J Atmos Ocean Technol 4:613-626.

Brabson BB, Palutikof JP (2000) Tests of the Generalized Pareto Distribution for predicting extreme wind speeds. J Appl Meteorol 39:1627-1640

Branford S, Coceal O, Thomas TG, Belcher SE (2011) Dispersion of a point-source release of a passive scalar through an urban-like array for different wind directions. Boundary-Layer Meteorol 139:367-394

Brasseur O (2001) Development and application of a physical approach to estimating wind gusts. Monthly Weather Review 129:5-25

Carneiro TC, Melo SP, Carvalho PCM, Braga AP de S (2016) Particle Swarm Optimization method for estimation of Weibull parameters: A case study for the Brazilian northeast region. Renew Energy 86:751759

Carta JA, Ramirez P, Velazquez S (2009) A review of wind speed probability distributions used in wind energy analysis Case studies in the Canary Islands. Renew Sust Energy Rev 13:933-955

Coceal O, Thomas TG, Castro IP, Belcher SE (2006) Mean flow and turbulence statistics over groups of urbanlike cubical obstacles. Boundary-Layer Meteorol 121:491-519

Coceal O, Dobre A, Thomas TG, Belcher SE (2007) Structure of turbulent flow over regular arrays of cubical roughness. J Fluid Mech 589: 375-409

Coceal O, Goulart EV, Branford S, Thomas TG, Belcher SE (2014) Flow structure and near-field dispersion in arrays of building-like obstacles. J Wind Eng Ind Aerodyn 125:52-68

D’Amico G, Petroni F, Prattico F (2014) Wind speed and energy forecasting at different time scales: A nonparametric approach. Physica A 406: 59-66

Datta D, Datta D (2013) Comparison of Weibull distribution and Exponentiated Weibull distribution based estimation of mean and variance of wind data. Int J Energy Informat Communicat 4(4):1-11

Efthimiou GC, Bartzis JG (2011) Atmospheric dispersion and individual exposure of hazardous materials. J Hazard Mater 188:375-383 
Efthimiou GC, Bartzis JG (2014) Atmospheric dispersion and individual exposure of hazardous materials. Validation and intercomparison studies. Int J Environ Pollut 55:76-85

Efthimiou G, Bartzis JG, Andronopoulos S, Sfetsos A (2011a) Air dispersion modelling for individual exposure studies. Int J Environ Pollut 302-316.

Efthimiou GC, Bartzis JG, Koutsourakis N (2011b) Modelling concentration fluctuations and individual exposure in complex urban environments J Wind Eng Ind Aerodyn 99:349-356

Efthimiou GC, Berbekar E, Harms F, Bartzis JG, Leitl B (2015) Prediction of high concentrations and concentration distribution of a continuous point source release in a semi-idealized urban canopy using CFDRANS modeling. Atmos Environ 100:48-56

Fischer R, Bastigkeit I, Leitl B, Schatzmann M (2010) Generation of spatio-temporally high resolved datasets for the validation of LES-models simulating flow and dispersion phenomena within the lower atmospheric boundary layer. CWE2010, 23-27 May 2010, Chapel-Hill, NC, USA

Francisco-Fernández M, Quintela-del-Río A (2013) Nonparametric analysis of high wind speed data. Clim Dyn 40:429. doi:10.1007/s00382-011-1263-2

Gupta AK, Nadarajah S (2004) Handbook of beta distribution and its applications, Marcel Dekker, New York.

He Y, Monahan AH, Jones CG, Dai A, Biner S, Caya D, Winger K (2010) Probability distributions of land surface wind speeds over North America. J Geophys Res 115

Hertwig D, Efthimiou GC, Bartzis JG, Leitl B (2012) CFD-RANS model validation of turbulent flow in a semi idealized urban canopy. J Wind Eng Indust Aerodyn 111:61-72

Holmes JD, Moriarty WW (1999) Application of the generalized Pareto distribution to extreme value analysis in wind engineering. J Wind Eng Indust Aerodyn 83:1-10

Indhumathy D, Seshaiah CV, Sukkiramathi K (2014) Estimation of Weibull parameters for wind speed calculation at Kanyakumari in India. Int J Innovat Res Sci Eng Technol 3:8340-8345

Janssen WD, Blocken B, van Hooff T (2014) Computational evaluation of pedestrian wind comfort and wind safety around a high-rise building in an urban area. 7th Intl. Congress on Env. Modelling and Software, 1519 June 2014, San Diego, CA, USA.

Karthikeya BR, Negi PS, Srikanth N (2016) Wind resource assessment for urban renewable energy application in Singapore. Renew Energy 87:403-414

Kidmo DK, Danwe R, Doka SY, Djongyang N (2015) Statistical analysis of wind speed distribution based on six Weibull methods for wind power evaluation in Garoua, Cameroon. Rev Energ Renouv 18:105-125

Kollu R, Rayapudi SR, Narasimham SVL, Pakkurthi KM (2012) Mixture probability distribution functions to model wind speed distributions. Int J Energy Environ Eng 3:27

Koutsourakis N, Hertwig D, Efthimiou GC, Venetsanos AG, Bartzis JG, Leitl B (2012) Evaluation of the ADREA-HF LES code for urban air quality assessment, using the CEDVAL LES wind tunnel database. 8th International Conference on Air Quality - Science and Application, 19-23 March 2012, Athens, Greece.

Kristensen L, Casanova M, Courtney MS, Troen I (1991) In search of a gust definition. Boundary Layer Meteorology 55:91-107

Masseran N, Razali AM, Ibrahim K (2013). The probability distribution model of wind speed over east Malaysia. Res J Appl Sci Eng Technol 6:1774-1779 
Men Z, Yee E, Lien FS, Chen DWY (2016) Short-term wind speed and power forecasting using an ensemble of mixture density neural networks. Renew Energy 87:203-211

Morgan EC, Lackner M, Vogel RM, Baise LG (2011) Probability distributions for offshore wind speeds. Energy Convers Manag 52:15-26

Nemeş CM (2013) Statistical analysis of wind speed profile: a case study from Iasi region, Romania. Int J Energy Eng 3:261-268

Odo FC, Offiah SU, Ugwuoke PE (2012) Weibull distribution-based model for prediction of wind potential in Enugu, Nigeria. Adv Appl Sci Res 3:1202-1208

Palutikof JP, Brabson BB, Lister DH, Adcock ST (1999) A review of methods to calculate extreme wind speeds. Meteorol Appl 6:119-132

Petkovic' D (2015) Adaptive neuro-fuzzy approach for estimation of wind speed distribution. Elect Power Energy Syst 73:389-392

Rozas-Larraondo P, Inza I, Lozano JA (2014) A method for wind speed forecasting in airports based on nonparametric regression. Weather Forecast 29:6:1332-1342

Sallis PJ, Claster W, Hernandez S (2011) A machine-learning algorithm for wind gust prediction. Comput Geosci 37:1337-1344

Sarkar A, Singh S, Mitra D (2011) Wind climate modeling using Weibull and extreme value distribution. Int J Eng Sci Technol 3:100-106

Simiu E, Heckert NA, Filliben JJ, Johnson SK (2001) Extreme wind load estimates based on the Gumbel distribution of dynamic pressures: an assessment. Struct Safety 23:221-229

Steinkohl C, Davis RA, Kluppelberg C (2010) Extreme value analysis of multivariate high frequency wind speed data. Working Paper. University of Munich and Columbia University.

van Donk SJ, Wagner LE, Skidmore EL, Tatarko J (2005) Comparison of the Weibull model with measured wind speed distributions for stochastic wind generation. Trans. ASAE 48:503-510

Waewsak J, Chancham C, Landry M, Gagnon Y (2011) An analysis of wind speed distribution at Thasala, Nakhon Si Thammarat, Thailand. J Sust Energy Environ 2:51-55

Yao YF, Thomas TG, Sandham ND, Williams JJR (2001) Direct numerical simulation of turbulent flow over a rectangular trailing edge. Theor Comput Fluid Dyn 14:337-358

Zhang J,Wang P, Zheng X (2013) A prediction model for simulating near-surface wind gusts. Eur Phys J E $36: 51$ 